\title{
Synthesis and Structure-Activity Analysis of New Phosphonium Salts with Potent Activity Against African Trypanosomes
}

Andrea Taladriz ${ }^{a \S}$, Alan Healy ${ }^{a \S}$, Eddysson J. Flores Pérez ${ }^{a \S}, V$ Vanessa Herrero García ${ }^{a}$ Carlos Ríos Martínez ${ }^{a}$, Abdulsalam A. M. Alkhaldi ${ }^{b \S}$, Anthonius A. Eze ${ }^{b}$, Marcel Kaiser $^{c, d}$, Harry P. de Koning ${ }^{b}$, Antonio Chana $^{e}$ and Christophe Dardonville ${ }^{a^{*}}$

a Instituto de Química Médica, IQM-CSIC, Juan de la Cierva 3, E-28006 Madrid, Spain.

${ }^{\mathrm{b}}$ Institute of Infection, Immunity and Inflammation, College of Medical, Veterinary and Life Sciences, University of Glasgow, Glasgow, United Kingdom

c Swiss Tropical and Public Health Institute, Socinstrasse, 57, CH-4002 Basel, Switzerland.

${ }^{\mathrm{d}}$ University of Basel, Petersplatz 1, CH-4003 Basel, Switzerland.

e Instituto de Química Física "Rocasolano", IQFR-CSIC. Serrano 119, E-28006 Madrid, Spain.

* Corresponding author. Tel.: +34 912587490; Fax.: +34 915644853; e-mail: dardonville@iqm.csic.es

$\S$ These authors contributed equally to this work.

\footnotetext{
$\$$ Abbreviations: CoMFA, comparative molecular field analysis; $\mathrm{EC}_{50}, 50 \%$ effective concentration; HAPT, high affinity pentamidine transporter; HAT, human African trypanosomiasis; HEK cells, human embryonic kidney cells; LAPT, low affinity pentamidine transporter; NBS, $N$-bromosuccinimide; PI, propidium iodide; PLS, partial least square; RMSD, root mean square deviations; SAR, structure activity relationship; SE, standard error; SI, selectivity index;
} 
Abstract. A series of 73 bisphosphonium salts and 10 monophosphonium salt derivatives were synthesized and tested in vitro against several wild type and resistant lines of Trypanosoma brucei (T. b. rhodesiense STIB900, T. b. brucei strain 427, TbAT1-KO, and TbB48). More than half of the compounds tested showed a submicromolar $\mathrm{EC}_{50}$ against these parasites. The compounds did not display any crossresistance to existing diamidine therapies, such as pentamidine. In most cases, the compounds displayed a good selectivity index versus human cell lines. None of the known $T$. $b$. brucei drug transporters were required for trypanocidal activity, although some of the bisphosphonium compounds inhibited the Low Affinity Pentamidine Transporter. It was found that phosphonium drugs act slowly to clear a trypanosome population, but that only a short exposure time is needed for irreversible damage to the cells. A Comparative Molecular Field Analysis Model (CoMFA) was generated to gain insights into the SAR of this class of compounds, identifying key features for trypanocidal activity.

Keywords: phosphonium salt, chemotherapy, protozoa, Trypanosoma brucei, sleeping sickness, CoMFA, Stucture activity relationships, mitochondria, cationic compound. 


\section{Introduction}

Human African trypanosomiasis (HAT or sleeping sickness) is a parasitic disease caused by infection with two subspecies of trypanosomes: Trypanosoma brucei gambiense and T. b. rhodesiense. HAT is endemic in 36 countries of sub-Saharan Africa and, together with the corresponding condition in domestic animals, is a major cause of suffering and poverty for the affected population. If left untreated, the disease is usually fatal. ${ }^{1}$ However, the treatment options are scarce as few drugs are available (i.e., suramin, pentamidine, melarsoprol, eflornithine, and recently, the combination therapy nifurtimox-eflornithine). These are far from being ideal due to ineffectiveness to some trypanosome species or stages of the infection, as well as to toxicity, a parenteral mode of administration and the emergence of resistance. ${ }^{2,3}$

The antitrypanosomal activity of benzyltriphenylphosphonium salts against $T$. brucei was first reported in 1979 by Kinnamon et al.. ${ }^{4}$ Some compounds were curative in a murine model of $T$. $b$. rhodesiense infection. However, the study of the potential of phosphonium salts as antiprotozoal agents was not followed up by any research group as shown by the lack of literature on this subject in the last 30 years. We recently regained interest in the antiparasitic activity of phosphonium salts with the discovery of a series of benzophenone-derived bisphosphonium salt derivatives that showed a marked antileishmanial activity in vitro. ${ }^{5}$ In Leishmania, the best compound of the series (i.e., 4,4'-bis((tri-n-pentylphosphonium)methyl)benzophenone dibromide) was found to target the mitochondria of the parasite, inhibiting complex II of the respiratory chain. ${ }^{5}$ Since some of these benzophenone compounds also showed interesting activity against $T . \quad b$. rhodesiense, ${ }^{6}$ we decided to prepare new derivatives to systematically examine the structure-activity relationship of antitrypanosomal phosphonium compounds. Hence, we synthesized 60 new phosphonium salt derivatives with 
variations in the following parts of the lead structure: (a) linker type, (b) linker length, (c) number of cations, (d) nature of the counterion, and (e) nature of the phosphonium groups substituents $\mathrm{R}_{1}, \mathrm{R}_{2}, \mathrm{R}_{3}$ (Chart 1 ).

Chart 1. General Structure of Benzophenone-derived Bisphosphonium Salt Derivatives with Antileishmanial and Antitrypanosomal Activity, ${ }^{5}$ and New Series Being Studied.

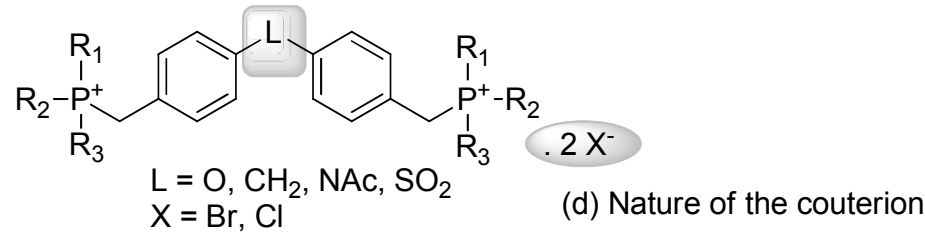

(a) Linker type

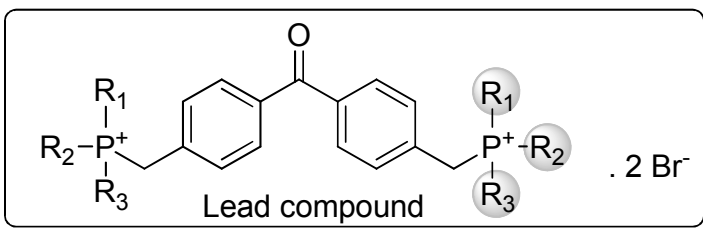

(b) Linker length

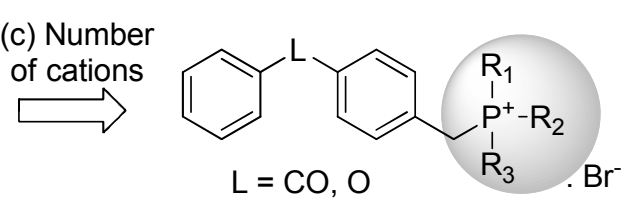

(e) Phosphonium substituents

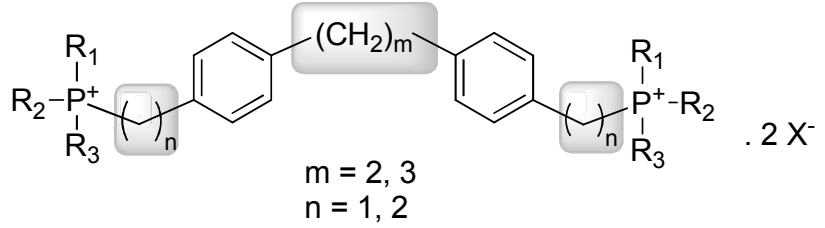

The compounds were tested for in vitro activity against $T$. $b$. rhodesiense (strain STIB900) and on a standard panel of $T$. b. brucei lines (s427, TbAT1-KO, and TbB48) with decreasing sensitivity to most diamidine and arsenic-based drugs due to loss of specific drug transporters (see experimental section for details). ${ }^{7,8}$ This is important because, like diamidines, most of the compounds described here are dications. In fact, the drug transporters may contribute positively to the selectivity of the compounds under development or, conversely, be a cause of drug resistance. ${ }^{9}$ Resistance to first line diamidines such as diminazene aceturate is linked to loss of these transporters ${ }^{10}$ and 
represents a genuine threat to the treatability of trypanosomiasis. ${ }^{2}$ Thus, it is essential to ascertain that no cross-resistance between new compounds and existing therapy will arise. In addition, we established the dynamics of the action of phosphonium salts on trypanosomes: trypanocidal or trypanostatic; fast action or slow; minimum time of exposure etc. Finally, we sought to understand the SAR behind this class of phosphonium compounds by performing a CoMFA analysis.

\section{Results}

Chemistry. The synthesis of the target compounds is based on the nucleophilic substitution of a (bis)halogenated precursor (i.e. linker) with a trisubstituted phosphine giving rise to the corresponding phosphonium salts (Scheme 1). Hence, the (bis)halogenated precursors must hold the different structural characteristics (i.e. linker type, linker length, number of cations, type of counterion) one wants to introduce in the lead structure.

The different halogenated linkers were synthetized as shown in Scheme 2. The 4,4'bisbromomethyl linkers $\mathbf{1 a},{ }^{6} \mathbf{1 b},{ }^{11} \mathbf{1 d},{ }^{12}$ and $\mathbf{1 e}^{13}$ were synthesized by $N$ bromosuccinimide (NBS) bromination of the 4,4'-dimethylphenyl precursors as previously reported. The linkers $1 \mathbf{c}$ and $\mathbf{2 c}$ were commercially available. The 4,4'bischloromethyl linker 3a was obtained by reaction of the corresponding 4,4'bisbromomethyl linker $\mathbf{1 a}$ with $\mathrm{BiCl}_{3}$ in anhydrous 1,2-dichloroethane. ${ }^{14}$ The diphenylethane (10f, 12f) and diphenylpropane (11g, 13g) linkers were synthesized as shown in Scheme 2. Friedel-Crafts acylation of diphenylethane and diphenylpropane (oxalyl chloride/ $\mathrm{AlCl}_{3}$ ) followed by decarbonylation in refluxing chlorobenzene gave 4 and $\mathbf{5},{ }^{15}$ respectively. The acyl chlorides were stirred in methanol at room temperature 
to yield the methyl esters 6 and 7 quantitatively. Lithium aluminium hydride reduction of 6 and 7 gave excellent yields of the diols 8 and 9. These were converted to the dibromide (10f, 11g) and dichloride (12f, 13g) using thionyl bromide and thionyl chloride, respectively. The synthesis of the 4,4'-bis(2-chloroethylphenyl) linkers $\mathbf{1 6 f}$ and $17 \mathrm{~g}$ started with Friedel-Crafts acylation of diphenylethane and diphenylpropane with chloroacetylchloride/ $\mathrm{AlCl}_{3}$. Reduction of $\mathbf{1 4}$ and 15 with triethylsilane in trifluoroacetic acid yielded the expected products $16 \mathbf{f}$ and $\mathbf{1 7 g}$, respectively.

Scheme 1. ${ }^{a}$ Synthesis of the Phosphonium Salt Derivatives ${ }^{b}$

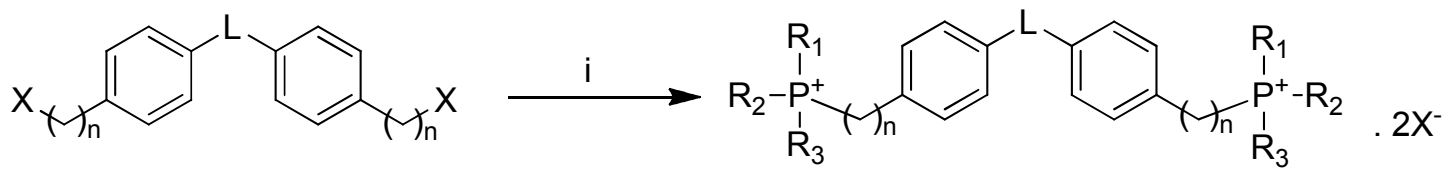

$\mathrm{n}=1, \mathrm{X}=\mathrm{Br}: \mathbf{1 a}, \mathbf{1 b}, \mathbf{1 c}, \mathbf{1 d}, \mathbf{1 e}, \mathbf{1 0 f}, \mathbf{1 1 g}$

$\mathrm{n}=1, \mathrm{X}=\mathrm{Cl}: \mathbf{3 a}, \mathbf{1 2 f}, \mathbf{1 3} \mathbf{g}$

$\mathrm{n}=2, \mathrm{X}=\mathrm{Cl}: \mathbf{1 6 f}, \mathbf{1 7 g}$ 18a-32a, 35a-45a, 47a-50a, 53a, 19b-c, 23b-e, $25 \mathrm{~b}-\mathrm{c}, 28 \mathrm{~b}-\mathrm{c}, 28 \mathrm{f}-\mathrm{g}, 33 \mathrm{~b}-\mathrm{c}, 34 \mathrm{~b}-\mathrm{c}, 38 \mathrm{~b}-\mathrm{c}, 43 \mathrm{~b}-\mathrm{c}$, 44b-c, 45b-g, 46b-c, 51b-c, 52b-c, 53b-c

$$
\mathrm{L}=\mathrm{CO}(\mathbf{a}), \mathrm{CH}_{2}(\mathbf{b}), \mathrm{O}(\mathbf{c}), \mathrm{SO}_{2}(\mathbf{d}), \mathrm{NAc}(\mathbf{e}),\left(\mathrm{CH}_{2}\right)_{2}(\mathbf{f}),\left(\mathrm{CH}_{2}\right)_{3}(\mathbf{g})
$$<smiles>CI(c1ccccc1)c1ccc(CBr)cc1</smiles>

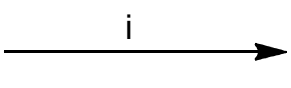

2a, 2c

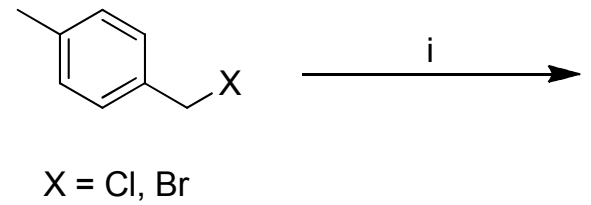<smiles>[R][P+]([R])([R2])Cc1ccc([Al](c2ccccc2)c2ccccc2)cc1</smiles>

$56 a, 56 c, 57 a, 57 c, 58 a-63 a$<smiles>[Y][PH2+](c1ccccc1)(c1ccccc1)c1ccc(C)cc1</smiles>

54: $\mathrm{X}=\mathrm{Cl}$

55: $\mathrm{X}=\mathrm{Br}$

\footnotetext{
${ }^{a}$ Reagents and conditions. (i) $\mathrm{R}_{1} \mathrm{R}_{2} \mathrm{R}_{3} \mathrm{P}$ (excess), DMF or toluene, $\Delta .{ }^{b}$ See Tables $1-4$ for substituents pattern.
} 
The bisphosphonium salts 18-53(a-e) were synthesized in good yields by reaction of the corresponding 4,4'-bischloromethyl- (3a, 12f, 13g), 4,4'-bisbromomethyl- (1a-1e, 10f, 11g), or 4,4'-bischloroethyl linker (16f, 17g) with an excess of commercially available trisubstituted phosphine in anhydrous DMF at $100{ }^{\circ} \mathrm{C}\left(150{ }^{\circ} \mathrm{C}\right.$ for $\mathbf{1 6 f}$ and $\left.\mathbf{1 7 g}\right)$ (Scheme 1). The monophosphonium salts $\mathbf{5 4}, \mathbf{5 5}, \mathbf{5 6 a}-\mathbf{6 3 a}$, and $\mathbf{5 6} \mathbf{c}-\mathbf{5 7} \mathbf{c}$ were prepared in the same way from 4-chloromethyltoluene, 4-bromomethyltoluene, 4bromomethylbenzophenone (2a), and 1-bromomethyl-4-phenoxybenzene (2c), respectively. A lower reaction temperature $\left(50{ }^{\circ} \mathrm{C}\right)$ was used with bis- and tris-2methoxyphenylphosphine (42a, 43a-c, and 61a) to avoid the formation of several byproducts. The salts were isolated by crystallization from the reaction mixture or by semi-preparative HPLC-MS for 64 and 65. Compounds 18a-24a, 26a, 28a-32a, 36a38a, 48a, and 49a were synthesized as reported earlier. ${ }^{5}$

Scheme 2. ${ }^{a}$ Synthesis of the Linkers.
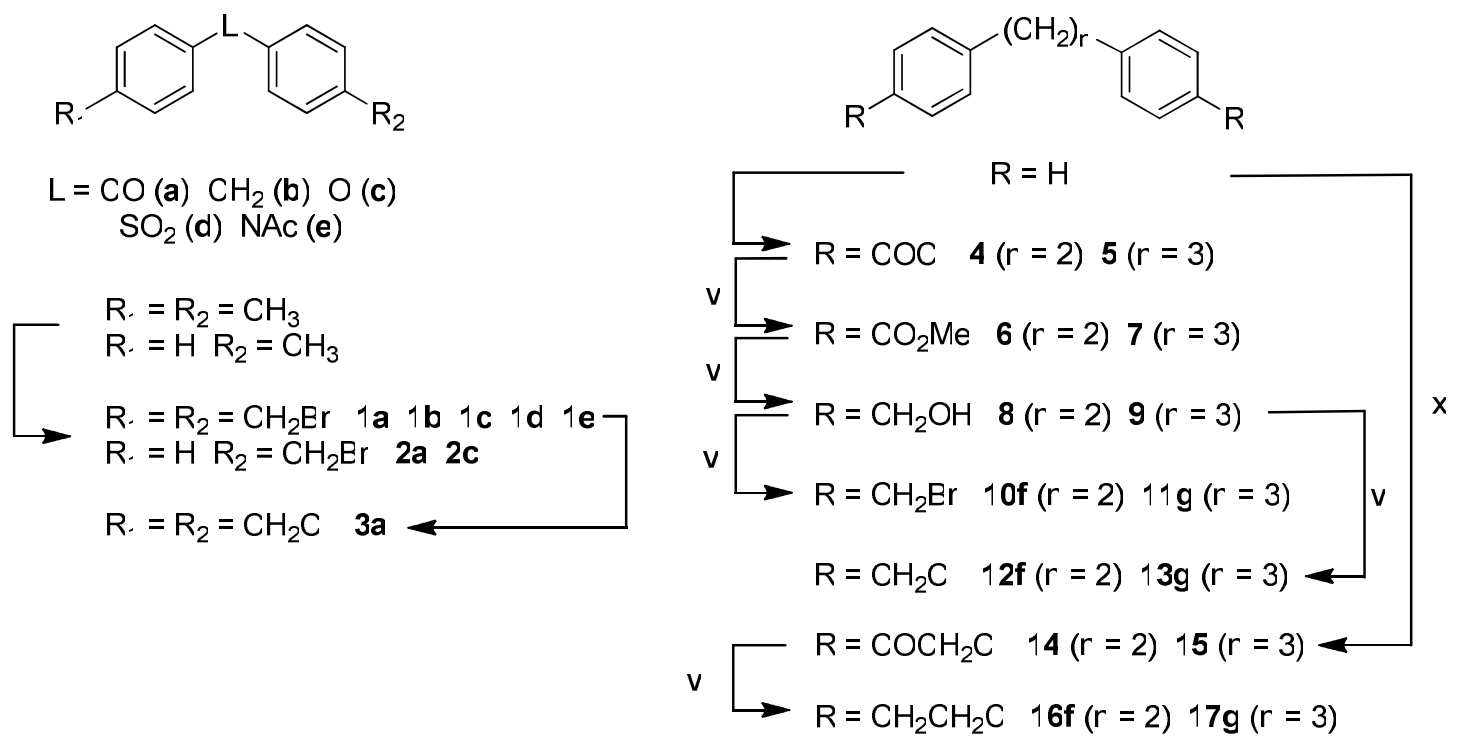

${ }^{a}$ Reagents and conditions. (i) NBS, $t \mathrm{BuOOH}, \mathrm{CCl}_{4}$, reflux, 20-52\%; (ii) $\mathrm{BiCl}_{3}, 1,2-$ dichloroethane, $\Delta, 60-72 \%$; (iii) 1) oxalyl chloride, $\mathrm{AlCl}_{3}, \mathrm{CH}_{2} \mathrm{Cl}_{2},-15{ }^{\circ} \mathrm{C}, 5 \mathrm{~h} ; 2$ ) 
chlorobenzene, reflux, 5h, 80-84\%; (iv) $\mathrm{MeOH}$, rt, quantitative; (v) $\mathrm{LiAlH}_{4}, \mathrm{THF}$, reflux, 18h, 90-94\%; (vi) thionyl bromide, $\mathrm{CH}_{2} \mathrm{Cl}_{2}, \mathrm{rt}, 2 \mathrm{~h}, 82-95 \%$; (vii) thionyl chloride, $\mathrm{CH}_{2} \mathrm{Cl}_{2}$, rt, 2h, 68-97\%; (viii) $\mathrm{Et}_{3} \mathrm{SiH}, \mathrm{CF}_{3} \mathrm{CO}_{2} \mathrm{H}, 0{ }^{\circ} \mathrm{C} \rightarrow 45^{\circ} \mathrm{C} \rightarrow \mathrm{rt}, 61-90 \%$; (ix) $\mathrm{ClCOCH}_{2} \mathrm{Cl}, \mathrm{AlCl}_{3}, \mathrm{CH}_{2} \mathrm{Cl}_{2}$, reflux, 3h, 94\%;

In Vitro Antitrypanosomal Activity. The new compounds were tested for in vitro activity against $T$. b. rhodesiense (strain STIB900) and a standard panel of Trypanosoma brucei lines (s427, TbAT1-KO, and T. b. B48) with decreasing sensitivity to diamidine drugs. It should be noted that the small differences in phosphonium compounds $\mathrm{EC}_{50}$ values observed between $T$. $b$. rhodesiense STIB900 and T. $b$. brucei s427 are most probably due to the slightly different assay procedures used in the two laboratories involved in this work. ${ }^{16}$ Alternatively, they could reflect the minor biochemical differences between the two strains used.

In general, compounds with three short alkyl substituents (Me, Et, or Pr) on the phosphonium cations were poorly active against both trypanosome species $\left(\mathrm{EC}_{50} \geq 10\right.$ $\mu \mathrm{M}$ ), whatever the linker was (Table 1: entries 1-6; Table 4: entries 7, 9). The activity was enhanced with the increase in chain length $(n$-octyl $\approx n$-hexyl $>c$-hexyl $\approx n$-pentyl $>n-\mathrm{Bu}>i$ - $\mathrm{Bu}$ ) reaching a maximum for 6- to 8-carbon substituents (Table 1, entries 718). Replacement of the alkyl substituents with phenyl rings increased the activity in the order: $\mathrm{R}_{1}, \mathrm{R}_{2}, \mathrm{R}_{3}=$ alkyl $<\left(\mathrm{R}_{1}, \mathrm{R}_{2}=\right.$ alkyl; $\left.\mathrm{R}_{3}=\mathrm{Ph}\right)<\left(\mathrm{R}_{1}=\right.$ alkyl; $\left.\mathrm{R}_{2}, \mathrm{R}_{3}=\mathrm{Ph}\right)($ Table 1: entries 19-32).

Since the best activities and selectivities were obtained with phenyl substituents, we decided to study whether the presence of substituents on the aromatic ring would influence the antitrypanosomal activity. As shown in Table 2, the best results were 
obtained with the 4-Me substituted phenyl rings (entries 16-25). Substitution pattern for the phenyl ring, in order of decreasing activity, was: 4-Me $>3-\mathrm{Me}>\mathrm{H}$ and 2-OMe $>4-$ $\mathrm{OMe}>4-\mathrm{Cl} \approx 4-\mathrm{F}>4-\mathrm{CF}_{3}$. Within this series, the order of decreasing activity as a function of the linker was: $\mathrm{O} \approx\left(\mathrm{CH}_{2}\right)_{3}>\left(\mathrm{CH}_{2}\right)_{2} \approx \mathrm{CH}_{2}>\mathrm{CO}>\mathrm{SO}_{2} \approx \mathrm{NAc}$.

Bioisosteric replacement of the phenyl substituents of the phosphonium cation by 2thienyl groups (Table 3, entries 1,2) or 1-naphthyl substituents (Table 3, entries 10-12) maintained the activity. On the contrary, the introduction of an oxygenated (2-furanyl) or amino (2-pyridyl) heterocycle (entries 3, 4) was detrimental to the activity, presumably because these are more polar and the substituents can engage in hydrogen bonds. Likewise, the replacement of one phenyl susbtitutent by a more polar phenylsulphonate or pentafluorophenyl ring abolished the trypanocidal activity (Table 3, entries 6-9).

Interestingly, removal of one of the phosphonium cations did not reduce the antitrypanosomal activity (Table 4). On the contrary, lower $\mathrm{EC}_{50}$ values were observed for 56a, 56c, 57c, 59a, and 62a compared with their bisphosphonium salt analogues (45a, 45c, 46c, 38a, and 25a, respectively). Regarding the substituents pattern, the same SAR as for the bisphosphonium analogues was observed (i.e., p-tolyl $>$ m-tolyl and $n$ hex $>i-\mathrm{Bu}>>\mathrm{Et}$ ). In this series, replacement of the carbonyl group by an oxygen linker enhanced the in vitro activity 20 to 40 -fold against $T$. b. rhodesiense (compare 56a vs 56c, 57a vs 57c). The diphenylether analogues $56 \mathbf{c}$ and $57 \mathbf{c}$ were the most active compounds of all the series with $\mathrm{EC}_{50}$ values in the low nanomolar range against wild type and resistant T. b. brucei lines (Table 4, entries 4 and 6), respectively. Despite their submicromolar and low micromolar cytotoxicity towards L6-cells and HEK-cells, respectively, both compounds still have a reasonable therapeutic window with a 
selectivity index (SI) vs L6-cells of 131 and 62, and of 1145 and 876 vs HEK-cells, respectively.

As far as the counterion is concerned, no significant difference in in vitro activity was observed between the bromide and the chloride salt of the compounds (Table 2, entries 16-22). In addition, a longer linker (i.e., ethylene instead of methylene linker) between the phosphonium cation and the central diphenyl core hardly affected the activity as shown by the nanomolar $\mathrm{EC}_{50} \mathrm{~s}$ of compounds 65 (73 nM vs $63 \mathrm{nM}$ for $\left.45 \mathrm{~g}\right)$ and 64 (27 $\mathrm{nM}$ vs $50 \mathrm{nM}$ for $\mathbf{4 5 f}$ ) (Table 5).

In most cases, the compounds displayed an acceptable selectivity index towards HEK cells $(>100)$. However, higher cytotoxicity was found with L6-cells, indicating a notable difference in susceptibility to phosphonium compounds between the two cell lines.

Cross-resistance and drug transport. Importantly, $\mathrm{EC}_{50}$ values were found to be statistically identical for the s427, TbAT1-KO and B48 strains in all cases (Student $t$ test; $\mathrm{P}>0.05$ ). This indicates that there is no cross resistance between the phosphonium cations and the crucial diamidine and melaminophenyl arsenical classes of trypanocides. However, the resazurin assay employed to generate $\mathrm{EC}_{50}$ values provides a single reading after an initial 48-h incubation period with test compound followed by a further $24 \mathrm{~h}$ in the additional presence of the dye. While this allows for high throughput, reproducible $\mathrm{EC}_{50} \mathrm{~S}$, it gives no information on how speedily the compounds act on the cells. The relationship between drug concentration and minimal exposure time is important as it has major implications for potential drug development: should the drug be required to be present in circulation for $48 \mathrm{~h}$ at a concentration $>\mathrm{EC}_{90}$ this would inevitably require a far higher dosage, and more frequent administration, than achieving 
such a dose for $2 \mathrm{~h}$. We thus conducted a series of experiments to assess differential action on the cell lines by a small selection of phosphonium salts. We used incubations with the viability reporter dye propidium iodide (PI), which gives a fluorescent signal upon cell entry and binding to nucleic acid. ${ }^{17}$ Figure 1 shows the effects on cellular integrity by $\mathbf{2 4 a}$, recorded in real-time for a period of $>8 \mathrm{~h}$. This revealed that, for all three strains only concentrations $\geq 3.3 \mu \mathrm{M}$ affected viability over the course of the experiment, despite $\mathrm{EC}_{50}$ values $\leq 0.20 \mu \mathrm{M}$. Yet, the speed at which the cell membrane integrity was compromised appeared to be slightly less in the diamidine resistant strains. Similar observations were made for $25 b, 25 c, 26 a, 35 a, 36 a, 38 a, 43 a, 43 c, 45 d, 45 e$, 47a, and 55 (see online Supporting Information). 
Fig. 1

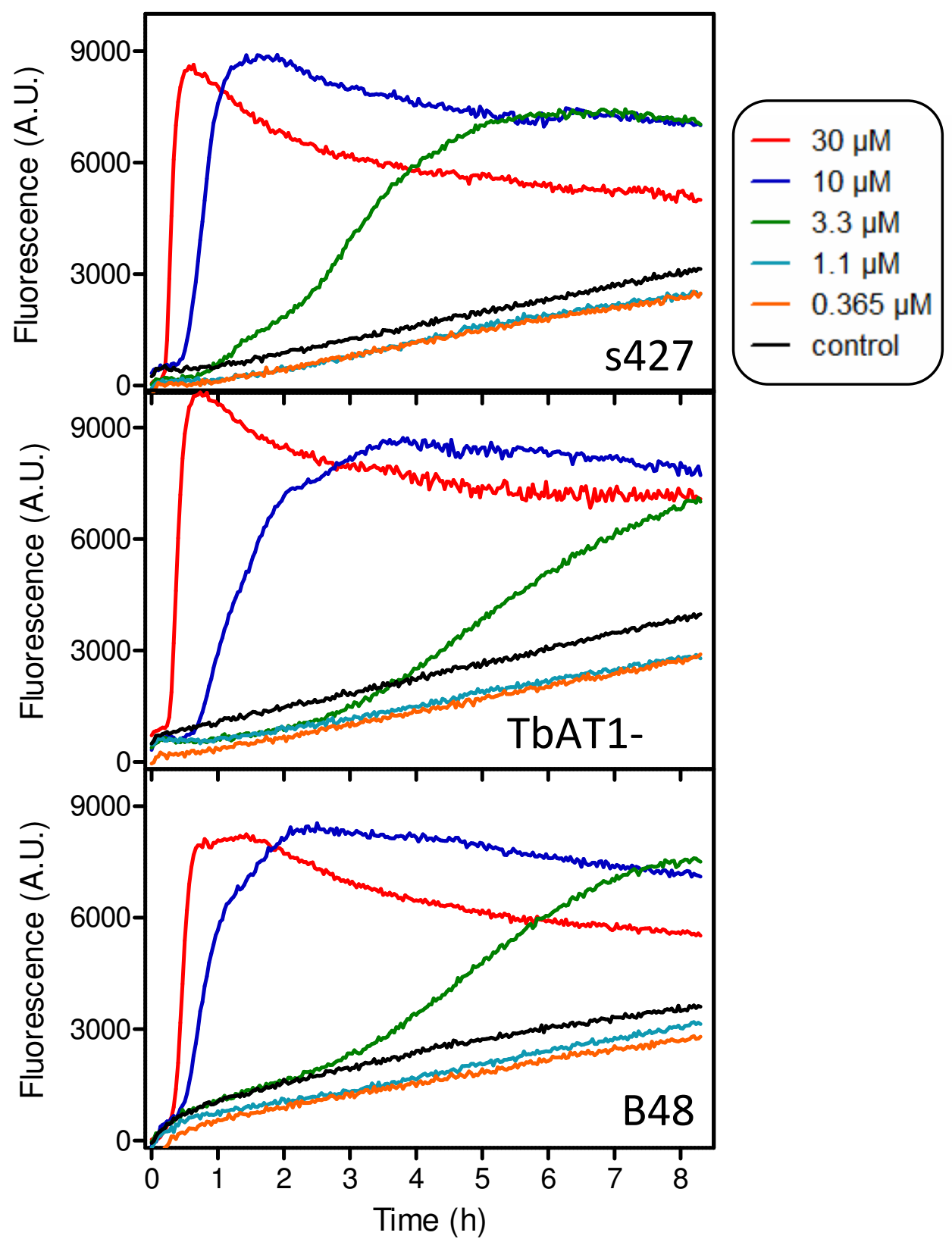

Figure 1. Real Time Monitoring of the Effects of 24a on Various Strains of T. $b$. brucei. The experiment was conducted with $5 \times 10^{5}$ cells/well in a 96-well format, with $200 \mu \mathrm{l} /$ well of HMI-9 medium containing $10 \%$ FBS and $9 \mu \mathrm{M}$ PI. Fluorescence was determined every 2 minutes for a total of 250 cycles; fluorescence was read using $544 / 620 \mathrm{~nm}$ filters for excitation and emission, respectively. Fluorescence values were determined in parallel for all three strains, using a single 96-well plate incubated in a Fluostar Optima fluorimeter at $37{ }^{\circ} \mathrm{C}$ and $5 \% \mathrm{CO}_{2}$. The slow increase in fluorescence in drug-free controls is attributable to the slow entry of PI into live trypanosomes. ${ }^{17}$ 
Given the very similar sensitivities, it must be deemed highly unlikely that the cellular entry of these cations depends critically on either of the two diamidine transporters (i.e., TbAT1/P2 aminopurine transporter and the High Affinity Pentamidine Transporter, HAPT1) that are known to be, by their absence, the cause of resistance in the strain B48. ${ }^{8}, 18$ We can speculate that, unlike diamidines, the positive charge(s) of the (bis)phosphonium compounds are highly dispersed and shielded by hydrophobic substituents, allowing trans-membrane diffusion at an appreciable rate. ${ }^{19}$ It is noted, in this context, that the anti-parasitic activity seems to be proportionate with the level of shielding, with methyl and ethyl substituents having virtually no effect (18a, 19a). Yet, some of the phosphonium compounds, such as $\mathbf{2 4 a}$ and $\mathbf{4 5 e}$ were good inhibitors of the diamidines transporters, especially of the Low Affinity Pentamidine Transporter (LAPT1) ${ }^{20}$ for which most of the compounds displayed higher affinity than the original substrate pentamidine (Table 6). As there was, however, no correlation between inhibition of the transporters and the antiparasitic activity, we conclude that the activity is not dependent on entry through any of the known drug transporters, with diffusion being the likely route of entry. 
Table 1. Antitrypanosomal Activity of Bisphosphonium Salts having Aliphatic and Phenyl Substituents.

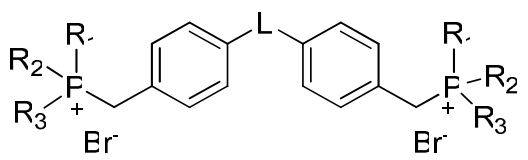

\begin{tabular}{|c|c|c|c|c|c|c|c|c|c|c|c|c|c|c|}
\hline Entry & Cmpd & $\mathbf{L}$ & $\mathbf{R}_{1}$ & $\mathbf{R}_{2}$ & $\mathbf{R}_{3}$ & T. b. rhod. ${ }^{a}$ & ${\text { Cytox. } \mathbf{L 6}^{b}}^{b}$ & T. b. brucei $\mathrm{WT}^{c}$ & TbAT1-KO $^{d}$ & $\mathbf{R F}^{e}$ & T.b. B48 & $\mathbf{R F}^{e}$ & Cytox. HEK $^{g}$ & $\mathbf{S I}^{h}$ \\
\hline & & & & & & \multicolumn{9}{|c|}{$\mathrm{EC}_{50}(\mu \mathrm{M})(\text { Selectivity index })^{i}$} \\
\hline & Melarsoprol & & & & & 0.0075 & & & & & & & & \\
\hline & Podophyllotoxin & & & & & & 0.012 & & & & & & & \\
\hline & Pentamidine & & & & & & & $0.008 \pm 0.002$ & $0.009 \pm 0.003$ & 1.1 & $0.567 \pm 0.086$ & 70.9 & & \\
\hline & Diminazene & & & & & & & $0.517 \pm 0.132$ & $2.39 \pm 0.84$ & 4.6 & $2.617 \pm 0.714$ & 5.1 & & \\
\hline 1 & 18a & $\mathrm{CO}$ & $\mathrm{Me}$ & $\mathrm{Me}$ & $\mathrm{Me}$ & 127 & $>173$ & $>100$ & $>100$ & & $>100$ & & $\mathrm{nd}^{\prime}$ & \\
\hline 2 & $19 \mathrm{a}$ & $\mathrm{CO}$ & Et & $\mathrm{Et}$ & Et & 77.8 & $>149$ & $>100$ & $>100$ & & $>100$ & & $>300$ & \\
\hline 3 & $19 b$ & $\mathrm{CH}_{2}$ & Et & Et & $\mathrm{Et}$ & 18.7 & 48.3 & $21.5 \pm 0.3$ & $18.5 \pm 0.4$ & 0.9 & $19.3 \pm 0.2$ & 0.9 & $>300$ & $>14$ \\
\hline 4 & $19 \mathrm{c}$ & $\mathrm{O}$ & Et & Et & $\mathrm{Et}$ & 31.9 & 101.6 & $36.5 \pm 3.5$ & $34.9 \pm 0.2$ & 1.0 & $36.6 \pm 0.6$ & 1.0 & $>300$ & $>8$ \\
\hline 5 & $20 \mathrm{a}$ & $\mathrm{CO}$ & $n-\operatorname{Pr}$ & $n-\operatorname{Pr}$ & $n$-Pr & 8.5 & $>130$ & $19.73 \pm 4.11$ & $27.15 \pm 3.82$ & 1.4 & $31.32 \pm 4.15$ & 1.6 & nd & \\
\hline 6 & $21 \mathrm{a}$ & $\mathrm{CO}$ & ${ }^{i} \operatorname{Pr}$ & ${ }^{i} \operatorname{Pr}$ & ${ }^{i} \operatorname{Pr}$ & 9.6 & $>130$ & $20.55 \pm 5.19$ & $33.41 \pm 6.20$ & 1.6 & $38.82 \pm 6.41$ & 1.9 & nd & \\
\hline 7 & $22 a$ & $\mathrm{CO}$ & $n$-Bu & $n$-Bu & $n-\mathrm{Bu}$ & $0.355(315)$ & 111.7 & $0.48 \pm 0.11$ & $1.32 \pm 0.46$ & 2.7 & $1.63 \pm 0.46$ & 3.4 & $>300$ & $>620$ \\
\hline 8 & $23 a$ & $\mathrm{CO}$ & ${ }^{i} \mathrm{Bu}$ & ${ }^{i} \mathrm{Bu}$ & ${ }^{i} \mathrm{Bu}$ & 1.46 & $>116$ & $2.62 \pm 0.69$ & $8.34 \pm 3.08$ & 3.2 & $6.30 \pm 1.39$ & 2.4 & $>300$ & $>110$ \\
\hline 9 & $23 b$ & $\mathrm{CH}_{2}$ & ${ }^{i} \mathrm{Bu}$ & ${ }^{i} \mathrm{Bu}$ & ${ }^{i} \mathrm{Bu}$ & $0.326(141)$ & 45.9 & $0.70 \pm 0.03$ & $0.88 \pm 0.10$ & 1.3 & $0.88 \pm 0.06$ & 1.3 & $>300$ & $>429$ \\
\hline 10 & $23 \mathrm{c}$ & O & ${ }^{i} \mathrm{Bu}$ & ${ }^{i} \mathrm{Bu}$ & ${ }^{i} \mathrm{Bu}$ & 1.62 & 51.1 & $0.59 \pm 0.06$ & $0.80 \pm 0.10$ & 1.3 & $1.06 \pm 0.34$ & 1.8 & $>300$ & $>507$ \\
\hline 11 & $23 d$ & $\mathrm{SO}_{2}$ & ${ }^{i} \mathrm{Bu}$ & ${ }^{i} \mathrm{Bu}$ & ${ }^{i} \mathrm{Bu}$ & 6.31 & $>111$ & $16.7 \pm 0.5$ & $13.6 \pm 2.2$ & 0.8 & $13.3 \pm 1.6$ & 0.8 & nd & \\
\hline 12 & $23 \mathrm{e}$ & NAc & ${ }^{\circ} \mathrm{Bu}$ & ${ }^{i} \mathrm{Bu}$ & ${ }^{i} \mathrm{Bu}$ & 11.44 & $>112$ & nd & nd & & nd & & nd & \\
\hline 13 & $24 a$ & $\mathrm{CO}$ & $n$-pentyl & $n$-pentyl & $n$-pentyl & $0.289(30)$ & 8.7 & $0.20 \pm 0.03$ & $0.17 \pm 0.01$ & 0.8 & $0.18 \pm 0.01$ & 0.9 & $66.8 \pm 4.9$ & 338 \\
\hline 14 & $25 a$ & $\mathrm{CO}$ & $n$-hex & $n$-hex & $n$-hex & $0.112(10)$ & 1.11 & $0.038 \pm 0.001$ & $0.047 \pm 0.007$ & 1.2 & $0.035 \pm 0.003$ & 0.9 & $16.6 \pm 0.7$ & 438 \\
\hline 15 & $25 b$ & $\mathrm{CH}_{2}$ & $n$-hex & $n$-hex & $n$-hex & $0.180(5)$ & 0.964 & $0.033 \pm 0.006$ & $0.033 \pm 0.005$ & 1.0 & $0.039 \pm 0.002$ & 1.2 & $18.1 \pm 2.6$ & 543 \\
\hline 16 & $25 c$ & $\mathrm{O}$ & $n$-hex & $n$-hex & $n$-hex & $0.109(9)$ & 1.03 & $0.037 \pm 0.004$ & $0.045 \pm 0.005$ & 1.2 & $0.041 \pm 0.002$ & 1.1 & $22.1 \pm 1.1$ & 593 \\
\hline 17 & $26 a$ & $\mathrm{CO}$ & $c$-hex & $c$-hex & $c$-hex & $0.197(166)$ & 32.7 & $0.14 \pm 0.03$ & $0.23 \pm 0.05$ & 1.6 & $0.25 \pm 0.04$ & 1.8 & $>300$ & $>2100$ \\
\hline 18 & $27 a$ & $\mathrm{CO}$ & $n$-octyl & $n$-octyl & $n$-octyl & $0.22(5)$ & 1.0 & $0.042 \pm 0.002$ & $0.061 \pm 0.001$ & 1.4 & $0.045 \pm 0.001$ & 1.1 & $27.0 \pm 1.4$ & 639 \\
\hline 19 & $28 \mathrm{a}$ & $\mathrm{CO}$ & $\mathrm{Me}$ & $\mathrm{Me}$ & $\mathrm{Ph}$ & 8.8 & $>139$ & $19.17 \pm 7.17$ & $28.77 \pm 5.62$ & 1.5 & $24.55 \pm 4.75$ & 1.3 & nd & \\
\hline 20 & $28 b$ & $\mathrm{CH}_{2}$ & $\mathrm{Me}$ & $\mathrm{Me}$ & $\mathrm{Ph}$ & 2.586 & $>142$ & $5.45 \pm 1.09$ & $4.60 \pm 0.80$ & 0.8 & $3.84 \pm 1.19$ & 0.7 & $>300$ & $>55$ \\
\hline 21 & $28 c$ & $\mathrm{O}$ & $\mathrm{Me}$ & $\mathrm{Me}$ & $\mathrm{Ph}$ & 3.827 & 84.5 & $7.22 \pm 0.16$ & $6.52 \pm 0.39$ & 0.9 & $6.15 \pm 0.89$ & 0.9 & $>300$ & $>40$ \\
\hline 22 & $28 \mathrm{f}$ & $\left(\mathrm{CH}_{2}\right)_{2}$ & $\mathrm{Me}$ & $\mathrm{Me}$ & $\mathrm{Ph}$ & $1.20(40)$ & 48.6 & $2.59 \pm 0.64$ & $2.62 \pm 0.16$ & 1.0 & $1.8 \pm 0.58$ & 0.5 & $>300$ & $>110$ \\
\hline 23 & $28 \mathrm{~g}$ & $\left(\mathrm{CH}_{2}\right)_{3}$ & $\mathrm{Me}$ & $\mathrm{Me}$ & $\mathrm{Ph}$ & $1.22(49)$ & 59.5 & $1.27 \pm 0.33$ & $1.37 \pm 0.11$ & 1.1 & $1.09 \pm 0.38$ & 0.9 & $>300$ & $>230$ \\
\hline 24 & 29a & $\mathrm{CO}$ & Et & Et & $\mathrm{Ph}$ & 2.9 & $>128$ & $8.59 \pm 2.16$ & $17.75 \pm 2.03$ & 2.1 & $15.17 \pm 3.72$ & 1.8 & $>300$ & $>35$ \\
\hline 25 & 30a & $\mathrm{CO}$ & $c$-hex & $c$-hex & $\mathrm{Ph}$ & $0.32(72)$ & 22.9 & $0.24 \pm 0.03$ & $0.26 \pm 0.04$ & 1.1 & $0.29 \pm 0.03$ & 1.2 & $>300$ & $>1270$ \\
\hline 26 & $31 \mathrm{a}$ & $\mathrm{CO}$ & $\mathrm{Me}$ & $\mathrm{Ph}$ & $\mathrm{Ph}$ & $0.656(177)$ & 115.9 & $1.53 \pm 0.38$ & $2.08 \pm 0.63$ & 1.4 & $2.05 \pm 0.38$ & 1.3 & $>300$ & $>200$ \\
\hline 27 & $32 \mathrm{a}$ & $\mathrm{CO}$ & Et & $\mathrm{Ph}$ & $\mathrm{Ph}$ & $0.554(>204)$ & $>113$ & $0.97 \pm 0.27$ & $2.10 \pm 0.54$ & 2.2 & $1.76 \pm 0.30$ & 1.8 & $>300$ & $>300$ \\
\hline 28 & $33 \mathrm{~b}$ & $\mathrm{CH}_{2}$ & $n$-Pr & $\mathrm{Ph}$ & $\mathrm{Ph}$ & $0.799(4.1)$ & 3.27 & $0.373 \pm 0.003$ & $0.40 \pm 0.01$ & 1.1 & $0.43 \pm 0.08$ & 1.1 & $>300$ & $>800$ \\
\hline 29 & $33 \mathrm{c}$ & $\mathrm{O}$ & $n$-Pr & $\mathrm{Ph}$ & $\mathrm{Ph}$ & $0.567(5)$ & 2.9 & $0.25 \pm 0.03$ & $0.28 \pm 0.01$ & 1.1 & $0.3 \pm 0.01$ & 1.2 & $233 \pm 31$ & 914 \\
\hline 30 & $34 \mathrm{~b}$ & $\mathrm{CH}_{2}$ & ${ }^{i} \operatorname{Pr}$ & $\mathrm{Ph}$ & $\mathrm{Ph}$ & $0.534(6.4)$ & 3.44 & $0.44 \pm 0.04$ & $0.40 \pm 0.03$ & 0.9 & $0.43 \pm 0.09$ & 1.0 & $>300$ & $>680$ \\
\hline 31 & $34 \mathrm{c}$ & $\mathrm{O}$ & ${ }^{i} \operatorname{Pr}$ & $\mathrm{Ph}$ & $\mathrm{Ph}$ & $0.585(8.6)$ & 5.0 & $0.33 \pm 0.02$ & $0.33 \pm 0.01$ & 1.0 & $0.33 \pm 0.01$ & 1.0 & $>300$ & $>900$ \\
\hline 32 & $35 \mathrm{a}$ & $\mathrm{CO}$ & $c$-hex & $\mathrm{Ph}$ & $\mathrm{Ph}$ & $0.43(155)$ & 65.8 & $0.30 \pm 0.03$ & $0.41 \pm 0.01$ & 1.4 & $0.33 \pm 0.03$ & 1.1 & $>300$ & $>1000$ \\
\hline
\end{tabular}

${ }^{a}$ T. b. rhodesiense STIB900 trypomastigotes; ${ }^{b}$ Rat skeletal myoblast L-6 cells; ${ }^{c}$ T. b. brucei s427 trypomastigotes; ${ }^{d}$ T. $b$. brucei knockout strain lacking a functional P2-transporter and resistant to diminazene aceturate ${ }^{18} ;{ }^{e}$ Resistance factor compared to $\mathrm{WT} ;{ }^{f}$ The B48 strain is a mutant derived from the 
TbAT1-KO strain with a nonfunctional High Affinity Pentamidine transporter (HAPT). This strain is resistant to diminazene, pentamidine and melaminophenyl arsenicals ${ }^{8} ;{ }^{g}$ Human Embryonic Kidney (HEK) cells; ${ }^{h}$ Selectivity index $=\left[\mathrm{EC}_{50}(\mathrm{HEK}\right.$ cells $) / \mathrm{EC}_{50}($ T. b . brucei WT) $] ;{ }^{i}$ Selectivity index $=\left[\mathrm{EC}_{50}\left(\mathrm{~L} 6_{-}\right.\right.$ cells) / $\mathrm{EC}_{50}$ (T. b. rhodesiense)]; ${ }^{j}$ Not determined. 
Table 2. Antitrypanosomal Activity of Bisphosphonium Salts having Substituted Phenyl Substituents ${ }^{a}$

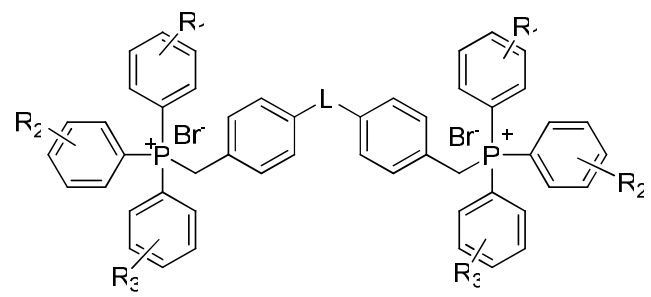

\begin{tabular}{|c|c|c|c|c|c|c|c|c|c|c|c|c|c|c|}
\hline Entry & Cmpd & $\mathbf{L}$ & $\mathbf{R}_{\mathbf{1}}$ & $\mathbf{R}_{2}$ & $\mathbf{R}_{3}$ & T. b. rhod. ${ }^{a}$ & Cytox. $\mathbf{L 6}^{b}$ & T. b. brucei $\mathbf{W T}^{c}$ & TbAT1-KO $^{d}$ & $\mathbf{R F}^{e}$ & T.b. B48 & $\mathbf{R F}$ & Cytox. HEK $^{g}$ & $\mathbf{S I}^{h}$ \\
\hline & & & & & & \multicolumn{9}{|c|}{$\mathrm{EC}_{50}(\mu \mathrm{M})(\text { Selectivity index })^{i}$} \\
\hline 1 & $36 \mathbf{a}$ & $\mathrm{CO}$ & $\mathrm{H}$ & $\mathrm{H}$ & $\mathrm{H}$ & $0.260(282)$ & 73.3 & $0.24 \pm 0.07$ & $0.36 \pm 0.07$ & 1.5 & $0.38 \pm 0.06$ & 1.6 & $>300$ & $>1250$ \\
\hline 2 & $37 \mathbf{a}$ & $\mathrm{CO}$ & $\mathrm{H}$ & $\mathrm{H}$ & 2-Me & $0.16(88)$ & 14.0 & $0.20 \pm 0.01$ & $0.24 \pm 0.03$ & 1.2 & $0.22 \pm 0.02$ & 1.1 & $214.3 \pm 9.5$ & 1098 \\
\hline 3 & $38 \mathbf{a}$ & $\mathrm{CO}$ & $\mathrm{H}$ & $\mathrm{H}$ & 4-Me & $0.26(62)$ & 16.3 & $0.180 \pm 0.002$ & $0.182 \pm 0.006$ & 1.0 & $0.183 \pm 0.005$ & 1.0 & $261.3 \pm 24.3$ & 1449 \\
\hline 4 & $38 \mathrm{~b}$ & $\mathrm{CH}_{2}$ & $\mathrm{H}$ & $\mathrm{H}$ & 4-Me & $0.200(22)$ & 4.47 & $0.24 \pm 0.04$ & $0.19 \pm 0.03$ & 0.8 & $0.18 \pm 0.02$ & 0.7 & $>300$ & $>1250$ \\
\hline 5 & $38 \mathrm{c}$ & $\mathrm{O}$ & $\mathrm{H}$ & $\mathrm{H}$ & 4-Me & $0.142(23)$ & 3.34 & $0.082 \pm 0.014$ & $0.077 \pm 0.002$ & 0.9 & $0.064 \pm 0.009$ & 0.8 & $240.3 \pm 6.7$ & 2930 \\
\hline 6 & 39a & $\mathrm{CO}$ & $4-\mathrm{Cl}$ & $4-\mathrm{Cl}$ & $4-\mathrm{Cl}$ & $0.41(18)$ & 7.2 & $0.94 \pm 0.14$ & $1.37 \pm 0.02$ & 1.5 & $0.95 \pm 0.13$ & 1.0 & $63 \pm 8$ & 67 \\
\hline 7 & $40 a$ & $\mathrm{CO}$ & $4-\mathrm{F}$ & $4-\mathrm{F}$ & $4-\mathrm{F}$ & $0.45(172)$ & 77.4 & $0.85 \pm 0.17$ & $2.10 \pm 0.03$ & 2.5 & $1.24 \pm 0.14$ & 1.5 & $>300$ & $>350$ \\
\hline 8 & $41 \mathrm{a}$ & $\mathrm{CO}$ & 4-OMe & 4-OMe & 4-OMe & $0.29(40)$ & 11.8 & $0.32 \pm 0.07$ & $0.52 \pm 0.02$ & 1.6 & $0.23 \pm 0.04$ & 0.7 & $172 \pm 7$ & 529 \\
\hline 9 & $42 a$ & $\mathrm{CO}$ & 2-OMe & $2-\mathrm{OMe}$ & $\mathrm{H}$ & $0.23(28)$ & 6.4 & $0.24 \pm 0.04$ & $0.32 \pm 0.02$ & 1.3 & $0.19 \pm 0.04$ & 0.8 & $107 \pm 15$ & 438 \\
\hline 10 & $43 a$ & $\mathrm{CO}$ & 2-OMe & $2-\mathrm{OMe}$ & $2-\mathrm{OMe}$ & $0.20(45)$ & 9.0 & $0.11 \pm 0.02$ & $0.16 \pm 0.01$ & 1.5 & $0.08 \pm 0.02$ & 0.7 & $131 \pm 17$ & 1175 \\
\hline 11 & $43 b$ & $\mathrm{CH}_{2}$ & 2-OMe & $2-\mathrm{OMe}$ & 2-OMe & $0.209(5)$ & 1.08 & $0.080 \pm 0.004$ & $0.074 \pm 0.003$ & 0.9 & $0.066 \pm 0.002$ & 0.8 & $204 \pm 13$ & 2561 \\
\hline 12 & $43 \mathrm{c}$ & $\mathrm{O}$ & 2-OMe & $2-\mathrm{OMe}$ & $2-\mathrm{OMe}$ & $0.153(8)$ & 1.27 & $0.056 \pm 0.011$ & $0.057 \pm 0.008$ & 1.0 & $0.045 \pm 0.004$ & 0.8 & $141 \pm 5$ & 2518 \\
\hline 13 & $44 a$ & $\mathrm{CO}$ & $4-\mathrm{CF}_{3}$ & $4-\mathrm{CF}_{3}$ & $4-\mathrm{CF}_{3}$ & 16.4 & 25.0 & $5.05 \pm 1.84$ & $8.51 \pm 1.98$ & 1.7 & $2.73 \pm 0.76$ & 0.5 & $>300$ & $>60$ \\
\hline 14 & $44 b$ & $\mathrm{CH}_{2}$ & $4-\mathrm{CF}_{3}$ & $4-\mathrm{CF}_{3}$ & $4-\mathrm{CF}_{3}$ & 1.72 & 7.52 & $0.23 \pm 0.06$ & $0.37 \pm 0.09$ & 1.6 & $0.28 \pm 0.04$ & 1.2 & $77.1 \pm 8.6$ & 338 \\
\hline 15 & $44 c$ & $\mathrm{O}$ & $4-\mathrm{CF}_{3}$ & $4-\mathrm{CF}_{3}$ & $4-\mathrm{CF}_{3}$ & 5.26 & 9.93 & $0.24 \pm 0.06$ & $0.32 \pm 0.05$ & 1.3 & $0.28 \pm 0.05$ & 1.2 & $174 \pm 23$ & 723 \\
\hline 16 & $45 \mathrm{a}$ & $\mathrm{CO}$ & 4-Me & 4-Me & 4-Me & $0.22(49)$ & 10.8 & $0.11 \pm 0.02$ & $0.018 \pm 0.01$ & 1.6 & $0.09 \pm 0.02$ & 0.8 & $141 \pm 10$ & 1266 \\
\hline 17 & $45 \mathrm{a}\left(\mathrm{Cl}^{-}\right.$salt $)$ & $\mathrm{CO}$ & 4-Me & 4-Me & 4-Me & $0.084(14.5)$ & 1.22 & $0.094 \pm 0.023$ & $0.065 \pm 0.002$ & 0.7 & $0.073 \pm 0.025$ & 0.8 & $24 \pm 1.2$ & 254 \\
\hline 18 & $45 b$ & $\mathrm{CH}_{2}$ & 4-Me & 4-Me & 4-Me & $0.099(24)$ & 2.33 & $0.080 \pm 0.002$ & $0.080 \pm 0.003$ & 1.0 & $0.074 \pm 0.001$ & 0.9 & $233 \pm 14$ & 2905 \\
\hline 19 & $45 \mathrm{f}$ & $\left(\mathrm{CH}_{2}\right)_{2}$ & 4-Me & 4-Me & 4-Me & $0.084(7.8)$ & 0.656 & $0.15 \pm 0.06$ & $0.15 \pm 0.02$ & 1.0 & $0.15 \pm 0.06$ & 1.0 & $32.4 \pm 2.4$ & 213 \\
\hline 20 & $\mathbf{4 5 f}\left(\mathrm{Cl}^{-}\right.$salt $)$ & $\left(\mathrm{CH}_{2}\right)_{2}$ & 4-Me & 4-Me & 4-Me & $0.050(33.8)$ & 1.69 & $0.058 \pm 0.026$ & $0.030 \pm 0.008$ & 0.5 & $0.045 \pm 0.018$ & 0.8 & $23.3 \pm 0.4$ & 404 \\
\hline 21 & $45 \mathrm{~g}$ & $\left(\mathrm{CH}_{2}\right)_{3}$ & 4-Me & 4-Me & 4-Me & $0.081(22)$ & 1.80 & $0.029 \pm 0.005$ & $0.049 \pm 0.007$ & 1.7 & $0.053 \pm 0.003$ & 1.8 & $25.8 \pm 1.7$ & 897 \\
\hline 22 & $45 \mathrm{~g}\left(\mathrm{Cl}^{-}\right.$salt $)$ & $\left(\mathrm{CH}_{2}\right)_{3}$ & 4-Me & 4-Me & 4-Me & $0.063(27.3)$ & 1.72 & $0.100 \pm 0.034$ & $0.066 \pm 0.005$ & 0.7 & $0.062 \pm 0.004$ & 0.6 & $12.7 \pm 0.5$ & 127 \\
\hline 23 & $45 \mathrm{c}$ & $\mathrm{O}$ & 4-Me & 4-Me & 4-Me & $0.170(14)$ & 2.34 & $0.024 \pm 0.005$ & $0.025 \pm 0.004$ & 1.0 & $0.021 \pm 0.001$ & 0.8 & $85.7 \pm 6.4$ & 3567 \\
\hline 24 & $45 d$ & $\mathrm{SO}_{2}$ & 4-Me & 4-Me & 4-Me & $0.173(127)$ & 22.0 & $0.23 \pm 0.01$ & $0.253 \pm 0.002$ & 1.1 & $0.27 \pm 0.01$ & 1.2 & $>300$ & $>1300$ \\
\hline 25 & $45 \mathrm{e}$ & NAc & 4-Me & 4-Me & 4-Me & $0.159(137)$ & 21.8 & $0.32 \pm 0.01$ & $0.30 \pm 0.01$ & 0.9 & $0.29 \pm 0.01$ & 0.9 & $>300$ & $>930$ \\
\hline 26 & $46 \mathrm{~b}$ & $\mathrm{CH}_{2}$ & 3-Me & 3-Me & 3-Me & $0.231(4)$ & 0.939 & $0.14 \pm 0.02$ & $0.14 \pm 0.01$ & 1.0 & $0.13 \pm 0.02$ & 0.9 & $72.6 \pm 7.1$ & 524 \\
\hline 27 & $46 c$ & $\mathrm{O}$ & $3-\mathrm{Me}$ & $3-\mathrm{Me}$ & $3-\mathrm{Me}$ & $0.488(1.9)$ & 0.942 & $0.057 \pm 0.013$ & $0.043 \pm 0.004$ & 0.7 & $0.088 \pm 0.024$ & 1.5 & $45.4 \pm 12.4$ & 792 \\
\hline
\end{tabular}

${ }^{a}$ See Table 1 for footnotes and reference drugs $\mathrm{EC}_{50}$ values. 
Table 3. Antitrypanosomal Activity of Bisphosphonium Salts having Aryl Groups or Heterocycle Substituents $^{a}$

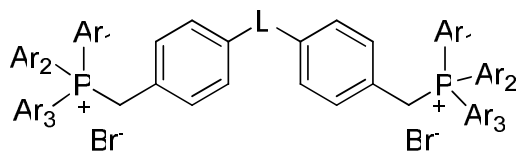

\begin{tabular}{|c|c|c|c|c|c|c|c|c|c|c|c|c|c|c|}
\hline Entry & Cmpd & $\mathbf{L}$ & $\mathbf{A \mathbf { r } _ { 1 }}$ & $\mathbf{A r _ { 2 }}$ & $\mathbf{A r _ { 3 }}$ & T. b. rhod. ${ }^{a}$ & Cytox. $\mathbf{L 6}^{b}$ & T. b. brucei $\mathrm{WT}^{c}$ & TbAT1-KO $^{d}$ & $\mathbf{R F}^{e}$ & T.b. B48 & $\mathbf{R F}^{e}$ & ${\text { Cytox. } \text { HEK }^{g}}$ & $\mathbf{S I}^{h}$ \\
\hline & & & & & & \multicolumn{9}{|c|}{$\mathrm{EC}_{50}(\mu \mathrm{M})(\text { Selectivity index })^{i}$} \\
\hline 1 & $36 a$ & $\mathrm{CO}$ & $\mathrm{Ph}$ & $\mathrm{Ph}$ & $\mathrm{Ph}$ & $0.260(282)$ & 73.3 & $0.24 \pm 0.07$ & $0.36 \pm 0.07$ & 1.5 & $0.38 \pm 0.06$ & 1.6 & $>300$ & $>1250$ \\
\hline 2 & $47 a$ & $\mathrm{CO}$ & 2-thienyl & 2-thienyl & 2-thienyl & $0.29(58)$ & 16.6 & $0.33 \pm 0.06$ & $0.52 \pm 0.03$ & 1.6 & $0.33 \pm 0.05$ & 1.0 & $260 \pm 21$ & 785 \\
\hline 3 & $48 \mathrm{a}$ & $\mathrm{CO}$ & 2-furanyl & 2-furanyl & 2-furanyl & 13.81 & 86.0 & $36.9 \pm 1.2$ & $33.8 \pm 1.2$ & 0.9 & $21.7 \pm 1.2$ & 0.6 & $\mathrm{nd}^{j}$ & \\
\hline 4 & $49 \mathrm{a}$ & $\mathrm{CO}$ & $\mathrm{Ph}$ & $\mathrm{Ph}$ & 2-pyridyl & $0.607(24)$ & 14.6 & $0.70 \pm 0.24$ & $1.43 \pm 0.41$ & 2.0 & $1.33 \pm 0.26$ & 1.9 & $>300$ & $>400$ \\
\hline 5 & $50 \mathrm{a}$ & $\mathrm{CO}$ & $\mathrm{Bn}$ & $\mathrm{Ph}$ & $\mathrm{Ph}$ & $0.48(67)$ & 32.0 & $0.81 \pm 0.12$ & $1.02 \pm 0.02$ & 1.3 & $1.03 \pm 0.22$ & 1.3 & $>300$ & $>370$ \\
\hline 6 & $51 b$ & $\mathrm{CH}_{2}$ & $\mathrm{Ph}$ & $\mathrm{Ph}$ & $\mathrm{C}_{6} \mathrm{~F}_{5}$ & 16.0 & $\mathrm{P}^{b}$ & $2.68 \pm 0.05$ & $3.79 \pm 0.09$ & 1.4 & $2.74 \pm 0.02$ & 1.0 & $>300$ & $>110$ \\
\hline 7 & $51 \mathrm{c}$ & $\mathrm{O}$ & $\mathrm{Ph}$ & $\mathrm{Ph}$ & $\mathrm{C}_{6} \mathrm{~F}_{5}$ & 12.8 & 5.35 & $23.2 \pm 3.1$ & $9.9 \pm 1.9$ & 0.4 & $6.68 \pm 1.65$ & 0.3 & $>300$ & $>10$ \\
\hline 8 & $52 \mathrm{~b}$ & $\mathrm{CH}_{2}$ & $\mathrm{Ph}$ & $\mathrm{Ph}$ & 3- $\left(\mathrm{SO}_{3} \mathrm{Na}\right) \mathrm{Ph}$ & $>92$ & $>92$ & $>100$ & $>100$ & & $>100$ & & $>300$ & \\
\hline 9 & $52 \mathrm{c}$ & $\mathrm{O}$ & $\mathrm{Ph}$ & $\mathrm{Ph}$ & 3- $\left(\mathrm{SO}_{3} \mathrm{Na}\right) \mathrm{Ph}$ & $>92$ & 90.5 & $>100$ & $>100$ & & $>100$ & & $>300$ & \\
\hline 10 & $53 a$ & $\mathrm{CO}$ & 1-naphthyl & 1-naphthyl & 1-naphthyl & $0.40(12)$ & 4.9 & $0.18 \pm 0.04$ & $0.339 \pm 0.005$ & 1.8 & $0.18 \pm 0.04$ & 1.0 & $92 \pm 4.0$ & 502 \\
\hline 11 & $53 \mathbf{b}$ & $\mathrm{CH}_{2}$ & 1-naphthyl & 1-naphthyl & 1-naphthyl & $0.299(12)$ & 3.43 & $0.14 \pm 0.02$ & $0.17 \pm 0.04$ & 1.3 & $0.14 \pm 0.01$ & 1.0 & $163 \pm 19$ & 1207 \\
\hline 12 & $53 \mathrm{c}$ & $\mathrm{O}$ & 1-naphthyl & 1-naphthyl & 1-naphthyl & $0.384(9)$ & 3.45 & $0.16 \pm 0.01$ & $0.14 \pm 0.01$ & 0.9 & $0.128 \pm 0.002$ & 0.8 & $114 \pm 16$ & 722 \\
\hline
\end{tabular}

${ }^{a}$ See Table 1 for footnotes and reference drugs $\mathrm{EC}_{50}$ values. ${ }^{b}$ Precipitate in solution.

Table 4. Antitrypanosomal Activity of Monophosphonium Salts ${ }^{a}$

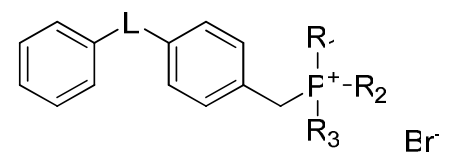

\begin{tabular}{|c|c|c|c|c|c|c|c|c|c|c|c|c|c|c|}
\hline Entry & Cmpd & $\mathbf{L}$ & $\mathbf{R}_{1}$ & $\mathbf{R}_{2}$ & $\mathbf{R}_{3}$ & T. b. rhod. ${ }^{a}$ & Cytox. $\mathbf{L 6}^{b}$ & T. b. brucei $\mathrm{WT}^{c}$ & TbAT1-KO $^{d}$ & $\mathbf{R F}^{e}$ & T.b. B48 & $\mathbf{R F}^{e}$ & Cytox. HEK $^{g}$ & $\mathbf{S I}^{h}$ \\
\hline & & & & & & \multicolumn{9}{|c|}{$\mathrm{EC}_{50}(\mu \mathrm{M})(\text { Selectivity index })^{i}$} \\
\hline 1 & 54 & & & & & $0.216(48)$ & 10.4 & $0.023 \pm 0.001$ & $0.024 \pm 0.002$ & 1.0 & $0.023 \pm 0.001$ & 1.0 & $>300$ & $>12500$ \\
\hline 2 & 55 & & & & & $0.364(35.5)$ & 12.9 & $0.043 \pm 0.001$ & $0.033 \pm 0.006$ & 0.8 & $0.022 \pm 0.000$ & 0.5 & $>300$ & $>7000$ \\
\hline 3 & $56 a$ & $\mathrm{CO}$ & 4-Me-Ph & 4-Me-Ph & 4-Me-Ph & $0.081(16.7)$ & 1.35 & $0.064 \pm 0.004$ & $0.012 \pm 0.002$ & 0.2 & $0.011 \pm 0.003$ & 0.2 & $32.5 \pm 3.6$ & 505 \\
\hline 4 & $56 \mathrm{c}$ & $\mathrm{O}$ & 4-Me-Ph & 4-Me-Ph & 4-Me-Ph & $0.002(131)$ & 0.263 & $0.015 \pm 0.002$ & $0.013 \pm 0.0006$ & 0.8 & $0.010 \pm 0.001$ & 0.7 & $17.6 \pm 1.2$ & 1145 \\
\hline 5 & $57 a$ & $\mathrm{CO}$ & 3-Me-Ph & 3-Me-Ph & 3-Me-Ph & $0.274(9.5)$ & 2.61 & $0.17 \pm 0.05$ & $0.15 \pm 0.03$ & 0.8 & $0.11 \pm 0.03$ & 0.7 & $48.2 \pm 2.4$ & 281 \\
\hline 6 & $57 \mathrm{c}$ & $\mathrm{O}$ & 3-Me-Ph & 3-Me-Ph & 3-Me-Ph & $0.014(62)$ & 0.870 & $0.028 \pm 0.002$ & $0.021 \pm 0.002$ & 0.8 & $0.017 \pm 0.0003$ & 0.6 & $24.5 \pm 2.3$ & 876 \\
\hline 7 & $58 \mathrm{a}$ & $\mathrm{CO}$ & ${ }^{i} \mathrm{Bu}$ & ${ }^{i} \mathrm{Bu}$ & ${ }^{i} \mathrm{Bu}$ & 4.48 & $>209$ & $5.9 \pm 0.7$ & $6.1 \pm 0.9$ & 1.0 & $6.28 \pm 0.85$ & 1.1 & $>300$ & $>50$ \\
\hline 8 & $59 a$ & $\mathrm{CO}$ & 4-Me-Ph & $\mathrm{Ph}$ & $\mathrm{Ph}$ & $0.267(29)$ & 7.72 & $0.15 \pm 0.03$ & $0.15 \pm 0.02$ & 1.0 & $0.10 \pm 0.01$ & 0.7 & $76.8 \pm 2.0$ & 504 \\
\hline 9 & $60 \mathrm{a}$ & $\mathrm{CO}$ & Et & Et & Et & $>254$ & $>254$ & $>100$ & $>100$ & & $>100$ & & $>300$ & \\
\hline 10 & $61 a$ & $\mathrm{CO}$ & 2-MeO-Ph & 2-MeO-Ph & 2-MeO-Ph & $0.376(10)$ & 3.97 & $0.21 \pm 0.05$ & $0.22 \pm 0.06$ & 1.1 & $0.15 \pm 0.03$ & 0.7 & $63.2 \pm 4.4$ & 306 \\
\hline 11 & $62 a$ & $\mathrm{CO}$ & $n$-hex & $n$-hex & $n$-hex & $0.103(15)$ & 1.61 & $0.011 \pm 0.004$ & $0.008 \pm 0.003$ & 0.7 & $0.022 \pm 0.011$ & 1.9 & $13.4 \pm 0.3$ & 1198 \\
\hline 12 & $63 a$ & $\mathrm{CO}$ & $\mathrm{Me}$ & $\mathrm{Me}$ & $\mathrm{Ph}$ & 16.94 & $>241$ & $42.7 \pm 6.2$ & $46.6 \pm 2.3$ & 1.1 & $45.8 \pm 12.8$ & 1.1 & $>300$ & $>7$ \\
\hline
\end{tabular}

${ }^{a}$ See Table 1 for footnotes and reference drugs $\mathrm{EC}_{50}$ values. 
Table 5. Antitrypanosomal Activity of Phosphonium Salts with Ethylene Linker ${ }^{a}$

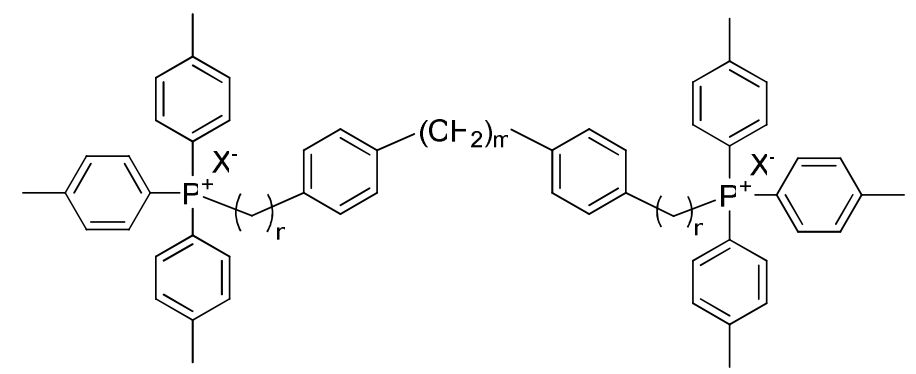

\begin{tabular}{|c|c|c|c|c|c|c|c|c|c|c|c|c|}
\hline Cmpd & Salt form & $\mathbf{m}$ & $\mathbf{n}$ & T. b. rhod. ${ }^{a}$ & Cytox. L6 $^{e}$ & T. b. brucei $\mathrm{WT}^{b}$ & TbAT1-KO $^{c}$ & $\mathbf{R F}$ & T.b. $\mathbf{B} 48^{d}$ & $\mathbf{R F}$ & Cytox. HEK $^{f}$ & $\mathrm{SI}^{8}$ \\
\hline & & & & \multicolumn{9}{|c|}{$\mathrm{EC}_{50}(\mu \mathrm{M})(\text { Selectivity index })^{i}$} \\
\hline $45 \mathrm{f}$ & $\mathrm{Br}^{-}$ & 2 & 1 & $0.084(7.8)$ & 0.656 & $0.15 \pm 0.06$ & $0.15 \pm 0.02$ & 1.0 & $0.15 \pm 0.06$ & 1.0 & $32.4 \pm 2.4$ & 213 \\
\hline $45 \mathrm{~g}$ & $\mathrm{Br}^{-}$ & 3 & 1 & $0.081(22)$ & 1.80 & $0.029 \pm 0.005$ & $0.049 \pm 0.007$ & 1.7 & $0.053 \pm 0.003$ & 1.8 & $25.8 \pm 1.7$ & 897 \\
\hline 64 & $\mathrm{Cl}^{-}$ & 2 & 2 & $0.027(40.3)$ & 1.09 & $0.125 \pm 0.027$ & $0.094 \pm 0.023$ & 0.8 & $0.089 \pm 0.017$ & 0.7 & $12.8 \pm 0.9$ & 103 \\
\hline 65 & $\mathrm{Cl}^{-}$ & 3 & 2 & $0.073(8.8)$ & 0.645 & $0.077 \pm 0.022$ & $0.043 \pm 0.009$ & 0.6 & $0.048 \pm 0.007$ & 0.6 & $5.6 \pm 0.2$ & 73 \\
\hline
\end{tabular}

${ }^{a}$ See Table 1 for footnotes and reference drugs $\mathrm{EC}_{50}$ values. 
Table 6. Affinity of Selected Phosphonium Compounds on the HAPT1 and LAPT1 Diamidine Transporters of T. b. brucei.

\begin{tabular}{ccc}
\hline Compound & $\mathbf{K}_{\mathbf{i}}$ value, HAPT1 & $\mathbf{K}_{\mathbf{i}}$ value, LAPT1 \\
& $(\mu \mathrm{M}, \pm \mathrm{SE})$ & $(\mu \mathrm{M}, \pm \mathrm{SE})$ \\
\hline Pentamidine & \\
$\mathbf{1 8 a}$ & $0.036 \pm 0.006$ & $56.2 \pm 8.3$ \\
$\mathbf{2 4 a}$ & $>250$ & $>250$ \\
$\mathbf{2 5 a}$ & $5.2 \pm 0.9$ & $2.2 \pm 0.4$ \\
$\mathbf{2 5 b}$ & $\mathrm{ND}{ }^{b}$ & $25 \pm 6$ \\
$\mathbf{2 5 c}$ & $\mathrm{ND}$ & $9.5 \pm 0.5$ \\
$\mathbf{2 6 a}$ & $53 \pm 13$ & $7.2 \pm 4.0$ \\
$\mathbf{3 5 a}$ & $\mathrm{ND}$ & $39 \pm 7$ \\
$\mathbf{3 6 a}$ & $\mathrm{ND}$ & $19 \pm 6$ \\
$\mathbf{3 8 b}$ & $\mathrm{ND}$ & $49 \pm 6$ \\
$\mathbf{4 5 e}$ & $\mathrm{ND}$ & $20 \pm 4$ \\
$\mathbf{5 5}$ & $9.2 \pm 1.3$ & $3.6 \pm 0.4$ \\
& $\mathrm{ND}$ & $25 \pm 11$ \\
\hline
\end{tabular}

Experiments were performed in triplicate, and on at least three separate occasions, as described in references $\left[{ }^{8,20}\right] .{ }^{a}$ Taken from $\operatorname{ref}\left[{ }^{20}\right] ;{ }^{b} \mathrm{ND}$, not determined.

Characterisation of the action of phosphonium salts on $T$. $b$. brucei. Figure 1 indicated that 24a only affected cellular viability during an 8-h experiment at concentrations $>15$-fold its $\mathrm{EC}_{50}$ value. In fact, numerous additional bisphosphonium salts were tested over $8 \mathrm{~h}$ on $T$. b. brucei s427 and typically affected parasite viability only at concentrations $70-120 \times \mathrm{EC}_{50}$ (see Supporting Information). The only 
exception was $\mathbf{5 5}$, with only concentrations $>700 \times \mathrm{EC}_{50}$ affecting parasite survival within this time frame. This indicates that mono-phosphonium salts may affect trypanosomes more slowly than the corresponding dications, probably because the single positive charge provides less driving force to cross inside-negative membranes. In fact, previous studies by Ross et al. ${ }^{19}$ have shown that lipophilic triphenylphosphonium dications are accumulated into mitochondria to a greater extent than their monocationic counterpart.

It is thus clear that, while monophosphonium and bisphosphonium compounds act potently on trypanosomes, their effect on cellular integrity is relatively slow. In order to assess the timing of the antitrypanosomal effect of phosphonium compounds we conducted manual cell counts on cultures incubated with various concentrations of test compound. We found that growth rates of T. b. brucei bloodstream forms were initially reduced upon incubation with phosphonium salts at concentrations of $\mathrm{EC}_{50}$ or slightly over, but typically recovered after $36 \mathrm{~h}$; only at concentrations $\geq 2 \times \mathrm{EC}_{50}$ did the cells not recover from the initial growth inhibition and started to die after $12 \mathrm{~h}$ (Figure 2A).

Although trypanosomes died only slowly on incubation with phosphonium compounds, only a relatively short exposure time was required. Figure $2 \mathrm{~B}$ shows that removal of $\mathbf{2 5 c}$ after a mere 30 min of incubation allowed for only very limited resumption of growth, reaching a maximum density of $9.4 \times 10^{5}$ cells $/ \mathrm{ml}$ at $48 \mathrm{~h}$ (i.e., $15 \%$ of the drug-free control). Removal of drug after $4 \mathrm{~h}$ did not lead to any resumption of growth at all, and the trypanosome population slowly dwindled over the next $44 \mathrm{~h}$. Continuous exposure to the drug resulted in sterilisation of the culture after approximately $30 \mathrm{~h}$. Highly similar results were obtained with a number of other compounds including $\mathbf{2 5 b}$ and $\mathbf{4 5 c}$. 
Fig. 2
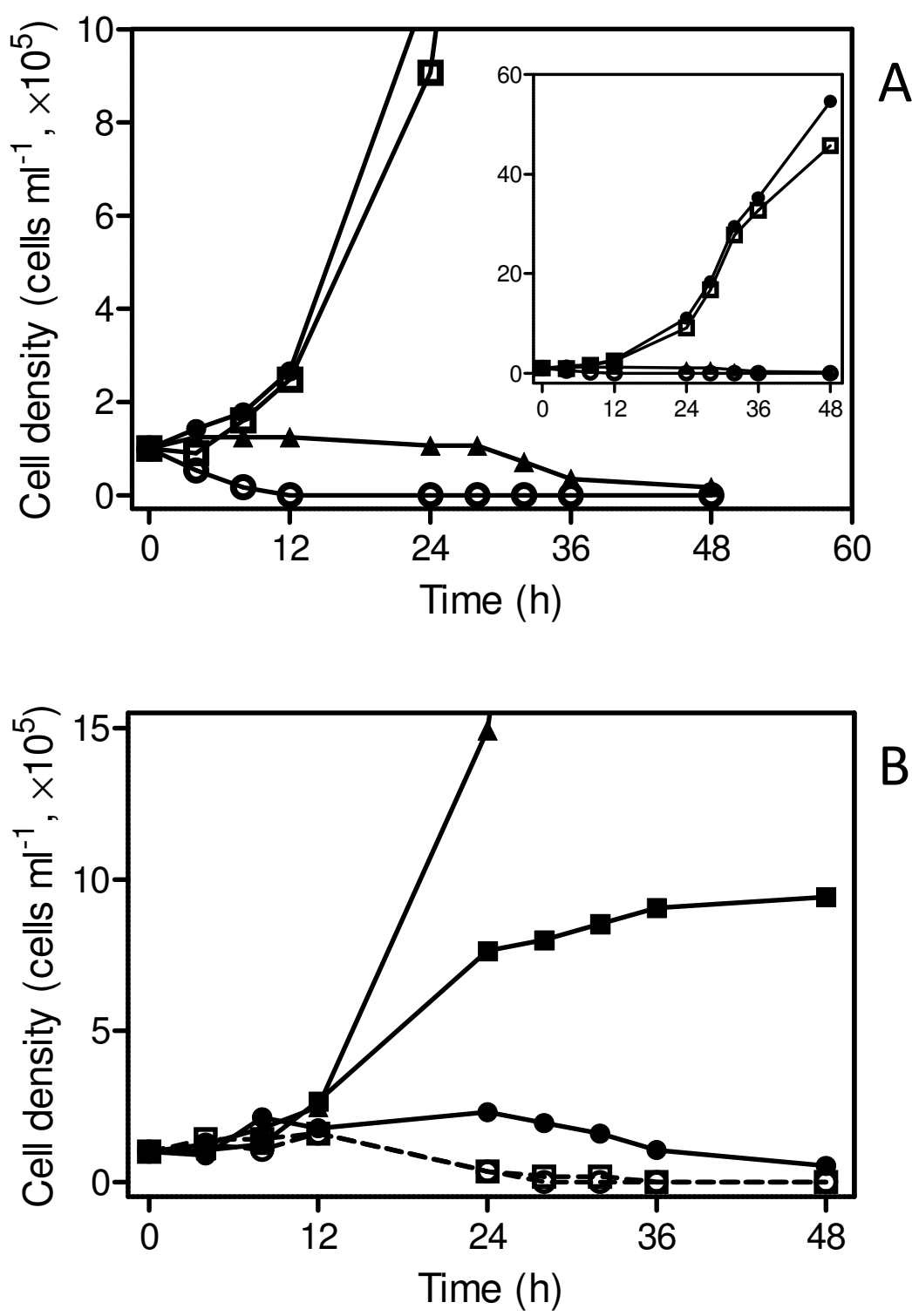

Figure 2. Effect of 25c on Cultures of T. b. brucei Bloodstream Forms. Cultures in the presence or absence of various concentrations of $\mathbf{2 5 c}$ were set up at a concentration of $10^{5}$ cells $/ \mathrm{ml}$. Microscopic cell counts were performed in triplicate using a haemocytometer and average values are shown. 
A. Cells were incubated in the continuous presence of $0.05 \mu \mathrm{M}(\square), 0.25 \mu \mathrm{M}(\mathbf{\Delta})$, or 1 $\mu \mathrm{M} \mathrm{25c}(\circ)$, or no compound (control, •). Inset: same data but showing the full growth over $48 \mathrm{~h}$ on a different scale.

B. Reversibility of growth inhibition by $0.25 \mu \mathrm{M} 25 \mathrm{c}$ was determined by centrifugation $1100 \times g)$ after either $30 \min (\bullet, \square)$ or $4 \mathrm{~h}(\bullet, \circ)$ and replacement with drug-free fresh medium (closed symbols, solid lines) or fresh medium with $0.25 \mu \mathrm{M}$ 25c (open symbols, dashed lines). A control culture $\left(\boldsymbol{\Lambda}\right.$, no drug) reached a density of $6 \times 10^{6}$ cells $/ \mathrm{ml}$ after $48 \mathrm{~h}$, as in panel A.

CoMFA Models. In order to gain insights into the SAR of this class of phosphonium salts, we have derived two main CoMFA models based on the $\mathrm{EC}_{50}$ values for $T . b$. rhodesiense and T. b. brucei, respectively (Tables 1-5). Both models are, at the least, able to discriminate activities and they possess a moderate to high predictive power. Nevertheless our hypothesis that both endpoints have a similar behaviour is confirmed by the modelling step. The two models are so alike that explaining both in details becomes redundant, consistent with identical modes of action for these closely related species. Therefore we are presenting here only the T. b. brucei model. For further reading about the $T$. $b$. rhodesiense model see the Supporting Information.

The training set (54 compounds) model statistics are fairly good: slope $0.8905, \mathrm{y}$ intercept $0.0466, \mathrm{r}^{2} 0.891, \mathrm{~F}$ 99.7. Standard validations on the training set were conducted in several ways: Leave One Out using SAMPLS algorithm ${ }^{21}$ (SAMpledistance Partial Least Squares), $q^{2}=0.43,3$ components Partial Least Squares (PLS) fitting; 10 groups Leave Several Out (LSO): $q^{2}=0.379,3$ components PLS fitting and bootstrapping: averaged $\mathrm{r}^{2} 0.856$ and averaged Standard Error (SE) was 0.343. Cross 
validation values were not actually promising so some suspicions of over fitting arose. However the test set statistics (Figure 3), a set never used in the modeling step, refuted this point. For T. b. brucei ( $\mathrm{pEC}_{50}$ exp. vs. $\mathrm{pEC}_{50}$ theor.), the model statistics are as follows: slope 0.7531 , intercept 0.0117 and $r^{2} 0.744$ (excluding outliers), or $r^{2} 0.67$ (including outliers). Such values fulfill the rules for predictive models proposed by Tropsha et $a .^{22}$ (i.e. $\mathrm{r}_{0}^{2} 0.743$ ), and can be considered as clearly predictive.

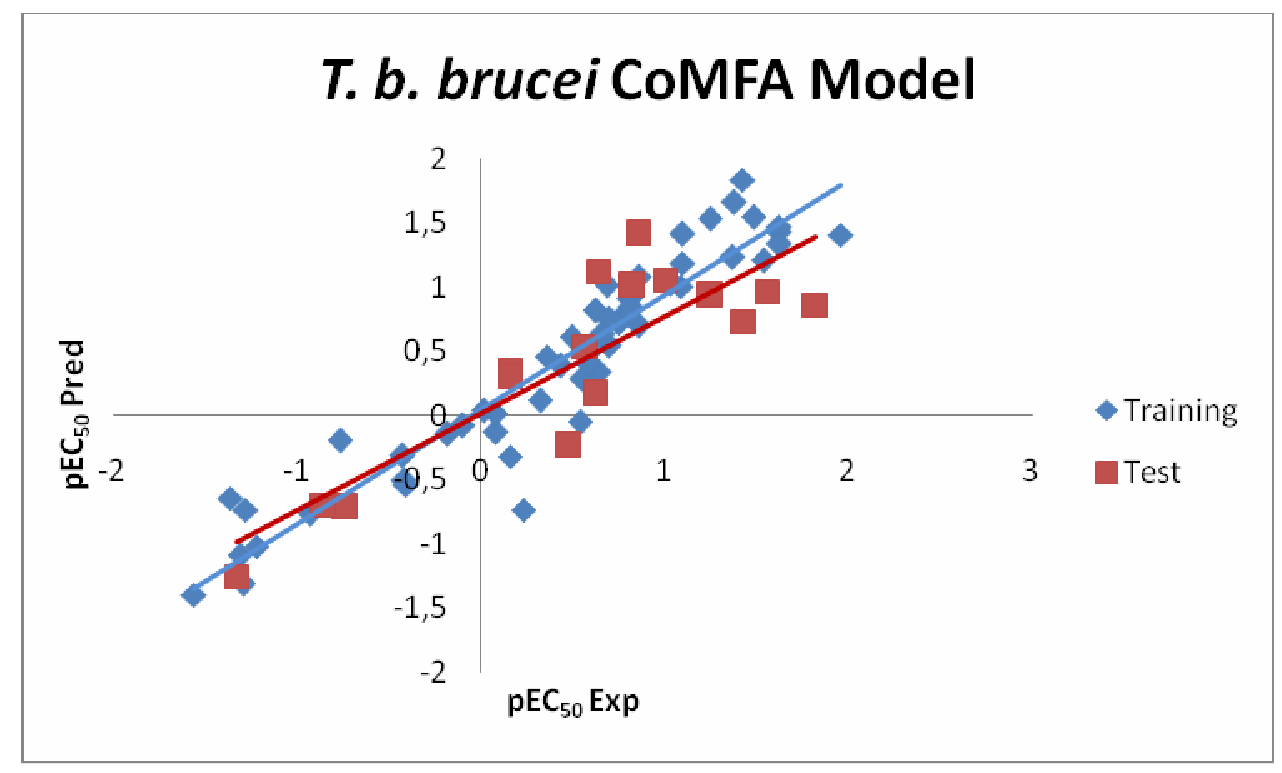

Figure 3. CoMFA Model for T. b. brucei. Plot of $-\log _{10}\left[\mathrm{EC}_{50}(\mu \mathrm{M})\right]_{\text {experimental }}$ vs. $\log _{10}\left[\mathrm{EC}_{50}(\mu \mathrm{M})\right]_{\text {predicted }}$ for T. b. brucei WT. Outliers are not shown; model statistics are commented in the text.

Leaving aside the good behavior of this model, a number of outliers were found within the test set: 48a, 52b, 52c and 63a. Compound 48a can be considered as an outlier due to its similar structure to $47 \mathbf{a}$, a rather active compound, whilst no other related structures were included. This fact clearly biased the model to predict a higher activity than it actually has. However, 48a was still predicted as inactive. 
52b and 52c were not included in the modeling step because their activities were not determined at the time of generating this model. These compounds are outliers because they are the only ones with neutral charge (i.e., presence of the 3-phenylsulphonate substituent) and no compound alike was included in the model. It should be noted that the counterions are not taken into account to generate the CoMFA models (see experimental part). Finally, 63a has clearly a lower activity than would be expected based on our model. This is probably due to the lack of lipophilic shielding around the phosphonium cation, reducing membrane permeation. For quantitative information see Table S2 in Supporting Information.

CoMFA fields derived from this model denote that the steric component is rather selective (Figure 4). Indeed the steric contribution to the CoMFA is 0.641. Bulk is strictly forbidden around the whole molecule except upon special positions on the phosphorous substituent. Such positions coincide with para and meta substitutions on 6membered aromatic rings which can also be easily occupied by long enough aliphatic chains. This fact explains why aliphatic chains are not active up to a certain number of carbons $(\mathrm{C} \leq 4)$; molecules with such small substituent do not fill the preferred volume (sse Figure 4A, compound 18a shown in blue) whilst they do not offer the essential lipophilic shielding to the phosphonium cation.

One issue derived from the good activities obtained for monocations is a remarkable loss of symmetry on the steric fields. While one end of the molecule is nearly surrounded by sterically unfavorable fields the distal end is virtually the opposite. Such assumption is coherent with the submicromolar activities found for dications with symmetric aromatic substitution (e. g. see Figure 4A, compound 41a shown in red). Here the favorable effect of bulk in one molecular end is compensated somehow by the same unfavorable bulk in the distal end. It is indeed the flexibility of aliphatic chains, 
able to avoid the restricted areas, the predominant effect on the nanomolar activities of compounds such as 25a-c (see Figure 4A, compound 25a showed in magenta) compared with 26a.

Electrostatic fields show that positive charge dispersion over the whole molecule favors the activity as we previously supposed. Here we see again a loss on the symmetry on the fields where a bit of negatively charged substituent, near the phosphonium (e.g., OMe, F...) and close to the more sterically impeded area, would favor the activity of these compounds. This is the case of compounds $43 \mathbf{b}$ and 43c bearing 2-methoxyphenyl substituents (see Figure 4B). In such cases this is the predominant effect confronted with the mere bulk offered by only aromatic substitution, such as in 50a. Surprisingly enough there is no other negative favorable area in the model. This is combined with a huge dispersion of the positive charge upon the molecule even surrounding the negative surfaces. We can deduce then a minor influence of localized electrostatic interactions, such as hydrogen bonds, with the phosphonium salts activities.

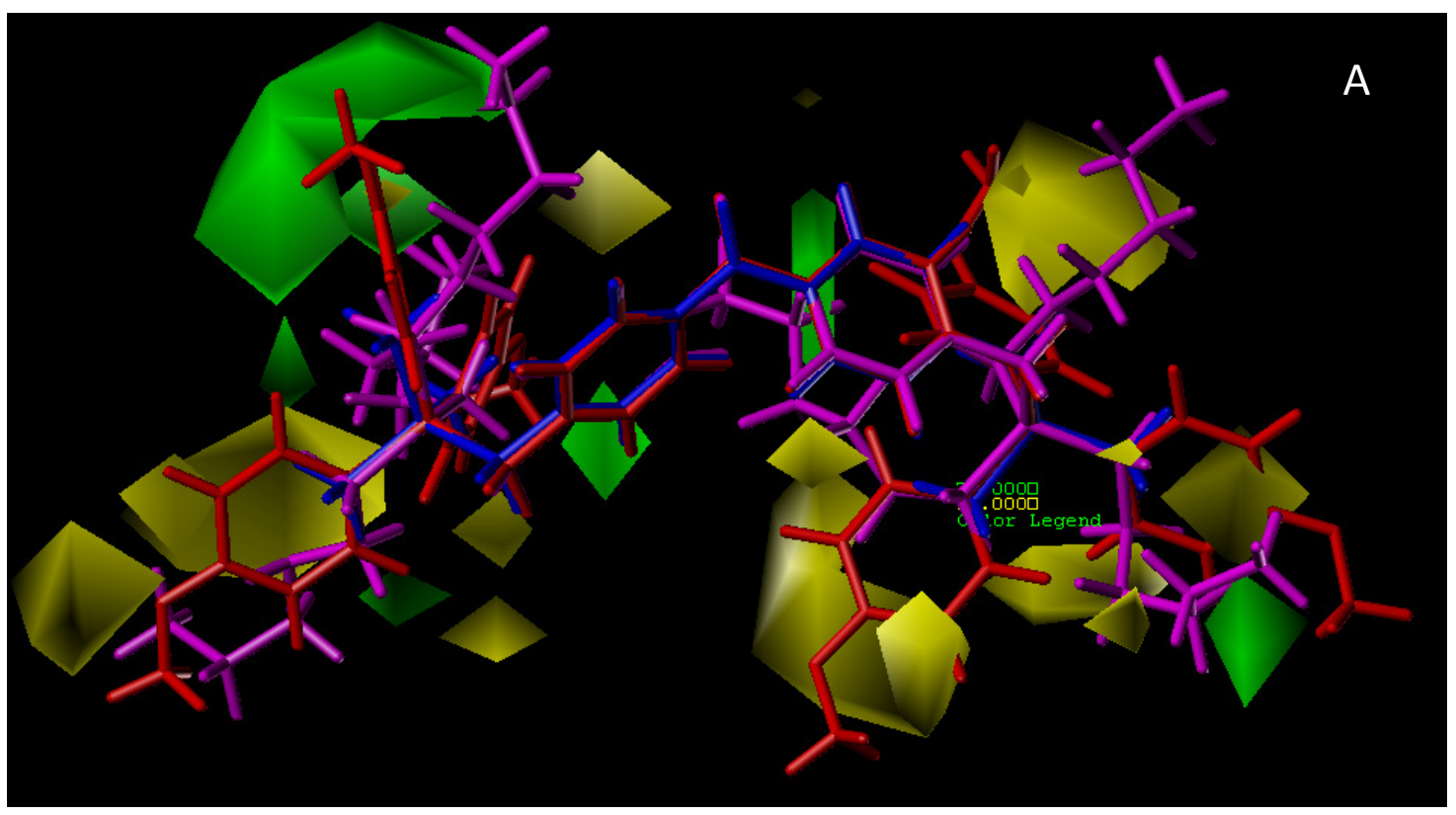




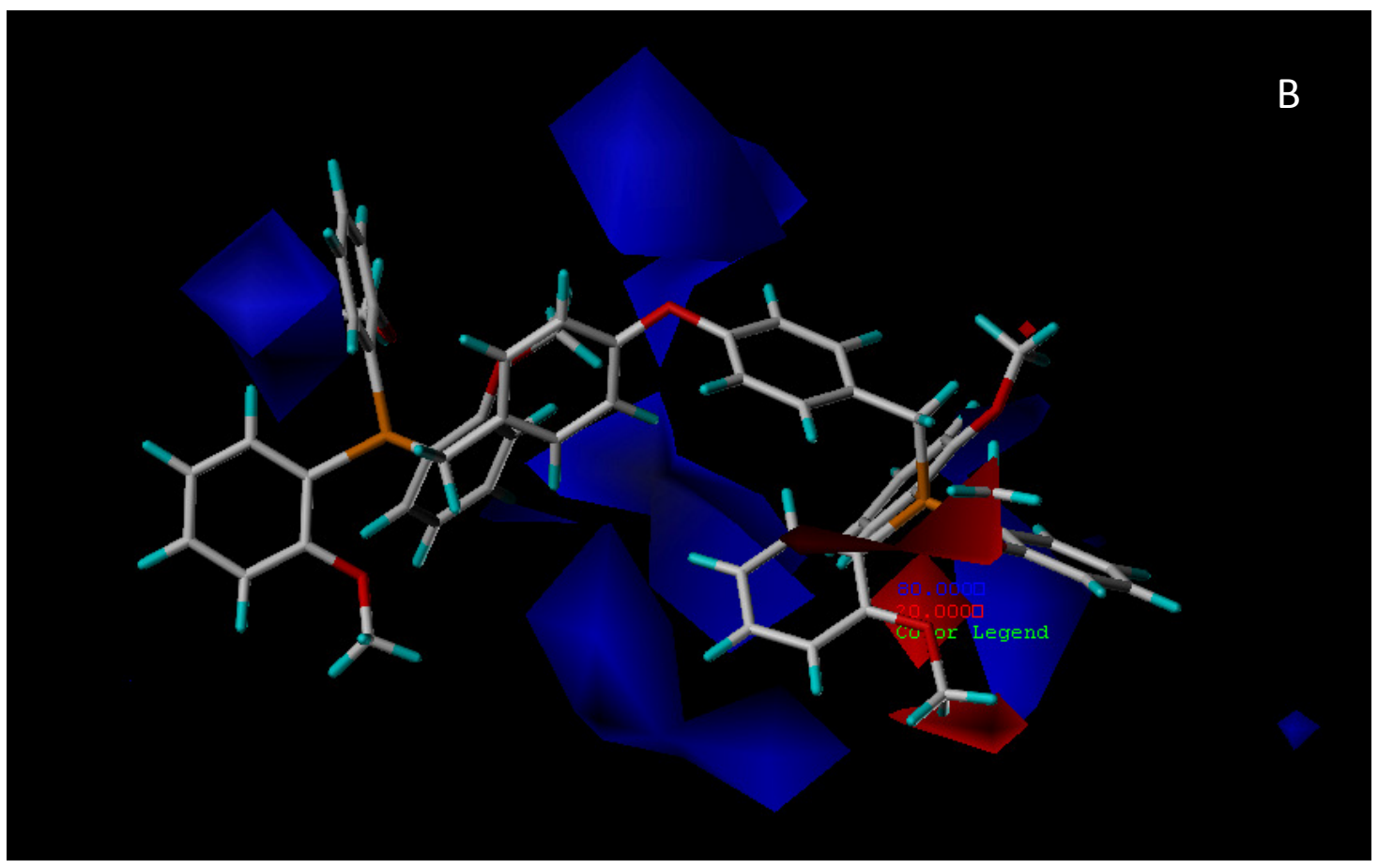

Figure 4. Steric and Electrostatic CoMFA Fields for T. b. brucei. A) Steric fields: regions where increasing the volume of the molecule favors antitrypanosomal activity are green; regions where increasing the volume decreases antitrypanosomal activity are yellow. Compounds 18a (blue), 25a (magenta), and 41a (red) are shown. B) Electrostatics field contours: indicate an increase of anti-T. brucei activity with increasing positive (blue) and negative (red) charge, respectively. Compound $43 \mathrm{c}$ is shown.

\section{Discussion}

Phosphonium salts bearing hydrophobic substituents are lipophilic cations with a delocalized charge. This affords specific properties to these molecules such as the capacity to cross biological membranes driven by electrical potential. Thus, they tend to accumulate in organelles with high membrane potential such as mitochondria. ${ }^{23}$ In Leishmania, several of the benzophenone-derived bisphosphonium salts reported here 
were found to target mitochondria of the parasite, a key to their leishmanicidal action. ${ }^{5}$ Ongoing studies on its mechanism of action in T. brucei point also to a mitochondrial target; for instance, phosphonium compounds strongly affected the mitochondrial membrane potential (unpublished results).

Interestingly, the SAR of the series of compounds presented here shows some similarities with the SAR against Leishmania. For instance, for compounds with homoalkyl groups, optimum activity was observed with 5- to 8-carbons, independently of the linker (Tables 1 and 4). In the same way, the combination of two phenyl groups and one alkyl substituent was enough to get submicromolar activity whereas the combination of one phenyl group and two short alkyl substituents (Me, Et) led to micromolar $\mathrm{EC}_{50}$. These observations are clearly supported by the CoMFA models which show that a minimum bulk around the phosphonium cations is favourable to the activity but this volume is also limited in size.

From these data, it is clear that a substantial level of lipophilic shielding around the phosphonium cation(s) is necessary to get high levels of activity against Trypanosoma and Leishmania species, presumably by increasing membrane permeation. No correlation was found between the antiparasitic activity and inhibition of the known $T$. brucei drug transporters (P2, HAPT1, and LAPT1). Although some bis-phosphonium compounds displayed surprisingly high affinity for the HAPT1 and LAPT1 transporters, most of the bis-phosphonium compounds would be considered too large to be a substrate (as opposed to be inhibitor) of such transporters (e.g., molecular weights range between 800 and 1300 compared with 340 for pentamidine). HAPT1 and LAPT1 were first described to transport a range of diamidines, ${ }^{20,}{ }^{24}$ which, like the bis-phosphonium compounds described here, are dications. Our CoMFA models show the importance of considerable positive charge dispersion over a large surface for increased activity. On 
balance, we conclude that, similar to a series of dicationic choline-derived compounds on which we recently reported, ${ }^{25}$ the antitrypanosomal activity is not dependent on entry through any of these transporters. In fact, it is highly probable that diffusion is the route of entry. This interpretation is much strengthened by the observation that substituents such as furan, pyridine and phenylsulphonate, which can engage in H-bonding, display significantly reduced antitrypanosomal activities, presumably because of impeded cellular penetration. The data presented in Table 4 clearly show that monophosphonium compounds have as good and often slightly better activity against trypanosomes than the corresponding symmetrical bis-phosphonium analogs. However, this improved activity is in many cases also coupled to somewhat increased toxicity against the mammalian cell lines employed. The redundancy of the second phosphonium group is consistent with the observation that longer, more flexible linkers (i.e., ethylene instead of methylene linker) between the phosphonium cation and the central diphenyl core hardly affected the antitrypanosomal activity. This observation is important because mono-phosphonium salts are smaller molecules with physicochemical properties (i.e., lower molecular weight, lower clogP) more likely to provide good pharmacokinetic behaviour in vivo compared with the bis-phosphonium counterparts.

In their 1979 paper, ${ }^{4}$ Kinnamon and Steck reported that the monophosphonium compound 54 (chloride salt) displayed 100\% curative activity (53 mg/kg sc) in a murine model of $T$. $b$. rhodesiense infection. The bromide analogue $\mathbf{5 5}$ was found to be less active in vivo than its chloride counterpart. However, no in vitro data was reported. The results of our in vitro screening are in agreement with these data as $\mathbf{5 4}$ displayed between submicromolar and nanomolar activities against $T . \quad b$. brucei and $T . b$. rhodesiense, respectively. 
The excellent in vitro antitrypanosomal activity observed with this set of phosphonium compounds confirms the potential of this class of molecules. We show that phosphonium compounds irreversibly inhibit trypanosome growth, leading to certain clearance of the population after only a minimum exposure time. This situation is similar to that of the highly successful class of diamidine trypanocides, which also require only a brief exposure time to ultimately kill the trypanosomes after $1-2$ days. ${ }^{26}$ This result is important for potential therapeutic applications, as dosage can be kept relatively low and a single administration may be sufficient, similar to the single administration of the diamidine diminazene aceturate that is the routine treatment for animal trypanosomiasis. ${ }^{2}$ In addition, the positive correlation with lipophilicity and thus membrane penetration should increase penetration into the central nervous system essential for treatment of late stage trypanosomiasis. ${ }^{3}$

\section{Conclusions}

The in vitro screening of a set of 83 phosphonium salt derivatives against African trypanosomes allowed the discovery of several trypanocidal compounds with nanomolar activities and adequate selectivities vs HEK and L6-cells. SAR studies showed that bulky substituents around the phosphonium cations (ie, either C5-C8 homoalkyl chains or phenyl rings) are necessary to get submicromolar activities, whereas the linkers have less influence on the antitrypanosomal activity. However, this statement is true as far as the bisphosphonium salts are concerned. On the contrary, the diphenylether linker seemed to be preferred in the case of monophosphonium compounds. In fact, 56c and 57c were the most active compounds of all the series with $\mathrm{EC}_{50}$ values in the low nanomolar range against wild type and resistant $T$. brucei lines. 
Phosphonium compounds displayed a very similar structure-activity relationship against T. brucei and Leishmania. ${ }^{5}$ This observation strongly suggests a similar mechanism of action against the two parasite species, and indicates that this class may have broad antiprotozoal activity. This is the subject of ongoing investigations in our laboratories.

Importantly, the screening reported here confirmed that there is no cross-resistance between the new compounds and existing diamidines and arsenical trypanocides, an essential condition for any new preclinical candidate.

Finally, the CoMFA models developed in this work should help in the design of new custom-made phosphonium derivatives with improved activity against African trypanosomes.

\section{Experimental section}

Chemistry. All dry solvents were purchased from Aldrich or Fluka in Sure/Seal bottles. All reactions requiring anhydrous conditions or an inert atmosphere were performed under a positive pressure of $\mathrm{N}_{2}$. All reactions were monitored by Thin Layer Chromatography (TLC) using silica gel $60 \quad \mathrm{~F}_{254}$ plates (Merck) or HPLC-MS. Chromatography was performed with Isolute SI prepacked columns. ${ }^{1} \mathrm{H}$ and ${ }^{13} \mathrm{C} \mathrm{NMR}$ spectra were recorded on a Bruker Advance 300 or Varian Inova 400 spectromether. Chemical shifts of the ${ }^{1} \mathrm{H}$ NMR spectra were internally referenced to the residual proton resonance of the deuterated solvents: $\mathrm{CDCl}_{3}(\delta 7.26 \mathrm{ppm}), \mathrm{D}_{2} \mathrm{O}(\delta 4.6 \mathrm{ppm}), \mathrm{CD}_{3} \mathrm{OD}(\delta$ $3.49 \mathrm{ppm})$ and DMSO ( $\delta 2.49 \mathrm{ppm}) . J$ values are given in Hz. Melting points were determined in open capillary tubes with a SMP3-Stuart Scientific apparatus or Mettler Toledo MP70 melting point system, and are uncorrected. All compounds are $>95 \%$ pure 
by HPLC or combustion analysis otherwise noted. Elemental analysis was performed on a Heraeus CHN-O Rapid analyser. Analytical results were within $\pm 0.4 \%$ of the theoretical values unless otherwise noted. Analytical HPLC-MS was run with an Xbridge C18-3.5 $\mu \mathrm{m}(2.1 \times 100 \mathrm{~mm})$ column on a Waters 2695 separation module coupled with a Waters Micromass ZQ spectromether using electrospray ionization $\left(\mathrm{ESI}^{+}\right)$. The following HPLC conditions were used: column temperature $=30{ }^{\circ} \mathrm{C}$, gradient time $=5 \mathrm{~min}, \mathrm{H}_{2} \mathrm{O} / \mathrm{CH}_{3} \mathrm{CN}(10: 90 \rightarrow 90: 10)\left(\mathrm{HCO}_{2} \mathrm{H} 0.1 \%\right)$, flow rate $=0.25$ $\mathrm{mL} / \mathrm{min}$, UV detection: diode array $(\lambda=190-400 \mathrm{~nm})$. Semi-preparative HPLC-MS was run with a SunFire Prep C18 $-5 \mu \mathrm{m}(19 \times 150 \mathrm{~mm})$ column on a Waters separation module (Waters 2545/SFO/2767) coupled to a Waters 3100 Mass Detector using ESI ${ }^{+}$. The fractions were collected with a Waters 2767 Sampler Manager.

1. General procedure for the synthesis of the bisphosphonium salts. A Kimax tube was charged with the appropriate bis-halogenated precursor (100 $\mathrm{mg}, \sim 0.28 \mathrm{mmol}$ ) and flushed with argon. Anhydrous DMF (3 mL) was added followed by the phosphine (1.12 mmol, 4 equiv). The tube was flushed with argon, stopped, and the reaction mixture was stirred at $100{ }^{\circ} \mathrm{C}$ for $20 \mathrm{~h}$. A higher temperature $\left(150{ }^{\circ} \mathrm{C}\right)$ and longer reaction time were necessary with the 4,4'-bischloroethyl linkers $\mathbf{1 6 f}$ and $\mathbf{1 7 g}$. Different workup procedures were used depending on whether the product precipitated from the reaction mixture or not. Workup I: the reaction was allowed to cool to room temperature and the precipitated product was collected by filtration, rinsed successively with toluene and $\mathrm{Et}_{2} \mathrm{O}$, and dried under vacuum. Workup II: the reaction mixture was transferred to a flask. Then, toluene $(10-20 \mathrm{~mL})$ was added to precipitate the product. The flask was stored in the fridge overnight. The supernatant was removed and the precipitate was rinsed with toluene. $\mathrm{Et}_{2} \mathrm{O}(10 \mathrm{~mL})$ was added and the precipitate was triturated with a spatula. The solid was collected, rinsed with $\mathrm{Et}_{2} \mathrm{O}$ and dried under vacuum. 
4,4'bis((triethylphosphonio)methyl)diphenylmethane dibromide (19b). The reaction was carried out in toluene following the general procedure with triethylphosphine and $\mathbf{1 b}$. The product was obtained as a white hygroscopic solid (107 $\mathrm{mg}, 65 \%$ ) following workup I procedure and recrystallization in DMF/Toluene. HPLC $=91 \%$ pure; $\operatorname{mp} 246-248{ }^{\circ} \mathrm{C} .{ }^{1} \mathrm{H}$ NMR $\left(300 \mathrm{MHz}, \mathrm{CDCl}_{3}\right) \delta 7.42(\mathrm{~d}, J=5.8,4 \mathrm{H}, \mathrm{Ar} H)$, $7.07(\mathrm{~d}, J=7.6,4 \mathrm{H}, \mathrm{Ar} H), 4.23(\mathrm{~d}, J=15.2,4 \mathrm{H}, \mathrm{PCH})_{2}, 3.88\left(\mathrm{~s}, 2 \mathrm{H}, \mathrm{PhCH} \mathrm{H}_{2} \mathrm{Ph}\right), 2.46$ (dt, $\left.J=20.5,7.6,12 \mathrm{H}, \mathrm{CH}_{2} \mathrm{CH}_{3}\right), 1.23\left(\mathrm{dt}, J=17.9,7.7,18 \mathrm{H}, \mathrm{CH}_{2} \mathrm{CH}_{3}\right) .{ }^{13} \mathrm{C} \mathrm{NMR}(75$ $\left.\left.\mathrm{MHz}_{,} \mathrm{CDCl}_{3}\right) \delta 156.9,131.9,123.2,119.9,25.6,25.0,12.3,11.6,6.0 . \mathrm{LRMS}_{(\mathrm{ES}}{ }^{+}\right) \mathrm{m} / \mathrm{z}$ $=429.39[(\mathrm{M}-\mathrm{H})]^{+}, 214.93\left[\mathrm{M}^{2+}, 100 \%\right]$. Anal. $\left(\mathrm{C}_{27} \mathrm{H}_{44} \mathrm{Br}_{2} \mathrm{P}_{2}\right)$ Calc: $\mathrm{C}, 54.93 ; \mathrm{H}, 7.51$; Br, 26.91. Found: C, 54.75; H, 7.72; $\mathrm{Br}, 26.20$.

4,4'-bis((trihexylphosphonio)methyl)benzophenone dibromide (25a). The reaction was carried out following the general procedure with trihexylphosphine and 1a for $48 \mathrm{~h}$ at $80{ }^{\circ} \mathrm{C}$. The product was obtained as a hygroscopic oily solid after workup II. Recrystallization from $\mathrm{MeOH} / \mathrm{Et}_{2} \mathrm{O}$ yielded a yellowish hygroscopic solid (27\%); HPLC $=89 \%$ pure. ${ }^{1} \mathrm{H}$ NMR $\left(300 \mathrm{MHz}, \mathrm{CDCl}_{3}\right) \delta 7.63(\mathrm{~d}, J=6.6,4 \mathrm{H}, \mathrm{Ar} H), 7.50(\mathrm{~d}, J=7.7$, 4H, $\mathrm{ArH}), 4.67\left(\mathrm{~d}, J=16.1,4 \mathrm{H} ; \mathrm{PCH}_{2} \mathrm{Ph}\right), 2.29\left(\mathrm{~m}, 12 \mathrm{H}, \mathrm{PCH}_{2} \mathrm{CH}_{2}\right), 1.6-1.1(\mathrm{~m}, 48 \mathrm{H}$, $\left.\left(\mathrm{CH}_{2}\right)_{4}\right), 0.84\left(\mathrm{~m}, 18 \mathrm{H}, \mathrm{CH}_{3}\right) .{ }^{13} \mathrm{C} \mathrm{NMR}\left(75 \mathrm{MHz}, \mathrm{CDCl}_{3}\right) \delta 194.9,136.5(\mathrm{~d}, J=3.4)$, $134.3(\mathrm{~d}, J=8.7), 130.6(\mathrm{~d}, J=4.8), 129.8(\mathrm{~d}, J=79.7), 31.1,30.6(\mathrm{~d}, J=14.7), 27.0$ (d, $J=44.4), 22.4,21.9(\mathrm{~d}, J=4.6), 19.2(\mathrm{~d}, J=46.1), 14.0 . \mathrm{LRMS}_{\left(\mathrm{ES}^{+}\right)} m / z 779.86$ $\left[(\mathrm{M}-\mathrm{H})^{+}\right], 390.11\left[\mathrm{M}^{2+}, 100 \%\right]$. ESI-HRMS $m / z 390.3233\left[\mathrm{M}^{2+}\right]\left(\mathrm{C}_{51} \mathrm{H}_{90} \mathrm{OP}_{2}\right.$ requires $390.3228)$.

4,4'-bis((trihexylphosphonio)methyl)diphenylmethane dibromide (25b). The reaction was carried out following the general procedure with trihexylphosphine and $\mathbf{1 b}$. The reaction was concentrated to ca. $1 \mathrm{~mL}$ to give a yellowish oil that was diluted with toluene $(10 \mathrm{~mL})$. Addition of $\mathrm{Et}_{2} \mathrm{O}(10 \mathrm{~mL})$ caused precipitation of an oily residue. The 
flask was stored in the fridge overnight. The supernatant was removed and the oily precipitate was rinsed with toluene. $\mathrm{Et}_{2} \mathrm{O}(10 \mathrm{~mL})$ was added to the oily precipitate, which was triturated with a spatula to yield $\mathbf{2 5 b}$ as a white solid (158.7 $\mathrm{mg}, 64 \%)$; mp $111-112{ }^{\circ} \mathrm{C}$ with previous softening (DMF/toluene); HPLC $>94 \%$ pure; ${ }^{1} \mathrm{H}$ NMR (300 $\left.\mathrm{MHz}, \mathrm{CDCl}_{3}\right) \delta 7.38(\mathrm{dd}, J=8.3,2.0,4 \mathrm{H}, \mathrm{Ar} H), 7.08(\mathrm{~d}, J=8.3,4 \mathrm{H}, \operatorname{Ar} H), 4.23(\mathrm{~d}, J=$ 15.1, 4H, $\mathrm{PCH}$ ), 3.89 (s, 2H, $\mathrm{PhCH} \mathrm{CH}_{2} \mathrm{Ph}$ ), 2.33 (br s, $12 \mathrm{H}, \mathrm{CH}_{2}$ ), 1.42 (br s, 24H, $\mathrm{CH}_{2}$ ), $1.23\left(\right.$ br s, $\left.24 \mathrm{H}, \mathrm{CH}_{2}\right), 0.85\left(\mathrm{t}, J=6.6,18 \mathrm{H}, \mathrm{CH}_{3}\right) .{ }^{13} \mathrm{C} \mathrm{NMR}\left(75 \mathrm{MHz}, \mathrm{CDCl}_{3}\right) \delta 140.8$, $130.5(\mathrm{~d}, J=4.9), 130.0(\mathrm{~d}, J=2.8), 126.5(\mathrm{~d}, J=8.8), 41.1,31.1,30.6(\mathrm{~d}, J=14.6)$,

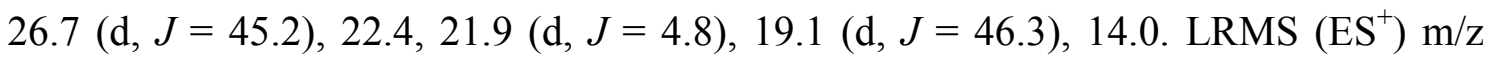
$765.87\left[(\mathrm{M}-\mathrm{H})^{+}\right], 383.04\left[\mathrm{M}^{2+}, 100 \%\right]$. ESI-HRMS $m / z 383.3357\left[\mathrm{M}^{2+}\right]\left(\mathrm{C}_{51} \mathrm{H}_{92} \mathrm{P}_{2}\right.$ requires 383.3332$)$.

\section{4,4'-bis((phenyldimethylphosphonio)methyl)diphenylethane dibromide}

(28f). The reaction was carried out following the general procedure with phenyldimethylphosphine (68.94 mg, $0.497 \mathrm{mmol})$ and $\mathbf{1 0 f}(73.2 \mathrm{mg}, 0.199 \mathrm{mmol})$. The reaction was concentrated under vacuum until the formation of an oily solid which was diluted with DMF. $\mathrm{Et}_{2} \mathrm{O}$ was added to precipitate the product as a white hygroscopic solid. The flask was allowed to stand in the freezer overnight. The solid was collected, rinsed with $\mathrm{Et}_{2} \mathrm{O}$ and dried under vacuum $(106.5 \mathrm{mg}, 98 \%$ ). HPLC $>95 \%$ pure; mp $>300{ }^{\circ} \mathrm{C} .{ }^{1} \mathrm{H}$ NMR $\left(300 \mathrm{MHz}, \mathrm{CDCl}_{3}\right) \delta 7.80(\mathrm{dd}, J=12.4,7.5,4 \mathrm{H}, \mathrm{Ar} H), 7.66(\mathrm{~m}, 2 \mathrm{H}$, $\operatorname{Ar} H), 7.57(\mathrm{~m}, 4 \mathrm{H}, \operatorname{Ar} H), 6.98(\mathrm{dd}, J=3.0,7.2,4 \mathrm{H}, \operatorname{Ar} H), 6.79(\mathrm{~d}, J=7.5,4 \mathrm{H}, \operatorname{Ar} H)$, $4.26(\mathrm{~d}, \mathrm{~J}=15.4,4 \mathrm{H}, \mathrm{PCH}), 2.72\left(\mathrm{~s}, 4 \mathrm{H} ; \mathrm{CH}_{2} \mathrm{CH}_{2}\right), 2.30\left(\mathrm{~d}, \mathrm{~J}=13.8,12 \mathrm{H}, \mathrm{CH}_{3}\right) .{ }^{13} \mathrm{C}$ $\operatorname{NMR}\left(75 \mathrm{MHz}, \mathrm{CDCl}_{3}\right) \delta 141.4(\mathrm{~d}, J=4.1), 134.6(\mathrm{~d}, J=1.8), 131.3(\mathrm{~d}, J=9.7), 130.2$ (d, $J=5.2), 130.0(\mathrm{~d}, J=12.4), 129.4(\mathrm{~d}, J=2.9), 125.4(\mathrm{~d}, J=9.1), 119.8(\mathrm{~d}, J=83.6)$, 36.9, $\left.31.2(\mathrm{~d}, J=48.0), 7.5(\mathrm{~d}, J=55.6) . \mathrm{LRMS}_{(\mathrm{ES}}{ }^{+}\right) \mathrm{m} / \mathrm{z}=483.00[(\mathrm{M}-\mathrm{H})]^{+}$. ESIHRMS $m / z 242.1241\left[\mathrm{M}^{2+}\right]\left(\mathrm{C}_{32} \mathrm{H}_{38} \mathrm{P}_{2}\right.$ requires 242.1219). 


\section{4,4'-bis((phenyldimethylphosphonio)methyl)diphenylpropane}

dibromide

(28g). The reaction was carried out following the general procedure with phenyldimethylphosphine (66 mg, $0.478 \mathrm{mmol})$ and $11 \mathrm{~g}(73.1 \mathrm{mg}, 0.191 \mathrm{mmol})$. The reaction was concentrated under vacuum until the formation of an oily solid which was diluted with acetone. $\mathrm{Et}_{2} \mathrm{O}$ was added to produce precipitation of the product as a dark brown hygroscopic solid (67.6 mg, $54 \%) . \mathrm{HPLC}=90 \%$ pure; ${ }^{1} \mathrm{H}$ NMR $(300 \mathrm{MHz}$, $\left.\mathrm{CDCl}_{3}\right) \delta 7.86-7.66(\mathrm{~m}, 7 \mathrm{H}, \mathrm{ArH}), 7.66-7.57(\mathrm{~m}, 3 \mathrm{H}, \mathrm{Ar} H), 6.98(\mathrm{t}, J=6.9,8 \mathrm{H}$, $\operatorname{Ar} H), 4.29(\mathrm{~d}, J=15.3,4 \mathrm{H}, \mathrm{PCH}), 2.58-2.45\left(\mathrm{~m}, 4 \mathrm{H}, \mathrm{PhCH}_{2} \mathrm{CH}_{2}\right), 2.37(\mathrm{~d}, J=13.7$, $\left.12 \mathrm{H}, \mathrm{CH}_{3}\right), 1.80\left(\mathrm{dd}, J=19.2,11.6,2 \mathrm{H}, \mathrm{PhCH}_{2} \mathrm{CH}_{2}\right) .{ }^{13} \mathrm{C} \mathrm{NMR}\left(75 \mathrm{MHz}, \mathrm{CDCl}_{3}\right) \delta$ $142.4(\mathrm{~d}, J=4.3), 134.6(\mathrm{~d}, J=3.2), 131.9(\mathrm{~d}, J=9.6), 130.5(\mathrm{~d}, J=5.5), 130.1(\mathrm{~d}, J=$ 12.2), $129.2(\mathrm{~d}, J=3.4), 125.3(\mathrm{~d}, J=9.4), 120.2(\mathrm{~d}, J=83.0), 35.1,32.3,31.3(\mathrm{~d}, J=$ 48.1), $7.8(\mathrm{~d}, J=55.5) . \mathrm{LRMS}\left(\mathrm{ES}^{+}\right) \mathrm{m} / \mathrm{z}=497.48[(\mathrm{M}-\mathrm{H})]^{+}, 248.95\left[\mathrm{M}^{2+}, 100 \%\right]$. ESIHRMS $m / z 249.1316\left[\mathrm{M}^{2+}\right]\left(\mathrm{C}_{33} \mathrm{H}_{40} \mathrm{P}_{2}\right.$ requires 249.1297).

\section{4,4'-bis((diphenyl-p-tolylphosphonio)methyl)diphenylether}

dibromide (38c). The reaction was carried out following the general procedure with diphenyl-p-tolylphosphine and 1c. The product was obtained as a white solid (187 mg, $66 \%$ ) following workup II procedure; mp $230{ }^{\circ} \mathrm{C}$ (decomp); HPLC $>97 \%$ pure; ${ }^{1} \mathrm{H}$ NMR $\left(300 \mathrm{MHz}, \mathrm{CDCl}_{3}\right) \delta 7.79-7.54(\mathrm{~m}, 28 \mathrm{H}, \mathrm{Ar} H), 7.42(\mathrm{dd}, J=7.9,2.8,4 \mathrm{H}, \mathrm{Ar} H)$, $7.08(\mathrm{dd}, J=8.6,2.4,4 \mathrm{H}, \mathrm{ArH}), 5.32(\mathrm{~d}, J=14.1,4 \mathrm{H}, \mathrm{PCH}), 2.45\left(\mathrm{~s}, 6 \mathrm{H}, \mathrm{CH}_{3}\right) .{ }^{13} \mathrm{C}$ NMR (75 MHz, $\left.\mathrm{CDCl}_{3}\right) \delta 156.8(\mathrm{~d}, J=4.0), 146.6(\mathrm{~d}, J=3.0), 135.0(\mathrm{~d}, J=2.7), 134.5$ (d, $J=9.9), 133.2(\mathrm{~d}, J=5.4), 131.1(\mathrm{~d}, J=12.9), 130.2(\mathrm{~d}, J=12.5), 122.4(\mathrm{~d}, J=8.6)$, $119.1(\mathrm{~d}, J=3.0), 118.1(\mathrm{~d}, J=85.7), 114.0(\mathrm{~d}, J=88.1), 30.1(\mathrm{~d}, J=47.3), 22.0$. LRMS $\left(\mathrm{ES}^{+}\right) \mathrm{m} / z 747.52\left[(\mathrm{M}-\mathrm{H})^{+}\right], 373.94\left[\mathrm{M}^{2+}, 100 \%\right.$ ]. ESI-HRMS $m / z 374.1536$ $\left[\mathrm{M}^{2+}\right]\left(\mathrm{C}_{52} \mathrm{H}_{46} \mathrm{OP}_{2}\right.$ requires 374.1506). 


\section{4,4'-bis((tri-4-methoxyphenylphosphonio)methyl)benzophenone}

dibromide (41a). The reaction was carried out following the general procedure with tris(4-methoxyphenyl)phosphine and $\mathbf{1 a}$ for $22 \mathrm{~h}$. The product was obtained as a white solid (214.5 mg, 74\%) following workup I procedure; mp 244.2-245 ${ }^{\circ} \mathrm{C}$; HPLC > 95\% pure. ${ }^{1} \mathrm{H}$ NMR $\left(300 \mathrm{MHz}, \mathrm{CDCl}_{3}\right) \delta 7.64(\mathrm{dd}, J=11.9,8.8,14 \mathrm{H}, \mathrm{Ar} H), 7.34(\mathrm{~d}, J=7.7$, 4H, $\operatorname{Ar} H), 7.25-7.19(\mathrm{~m}, 4 \mathrm{H}, \operatorname{Ar} H), 7.07(\mathrm{dd}, J=8.8,2.4,14 \mathrm{H}, \operatorname{Ar} H), 5.38(\mathrm{~d}, J=$ 15.2, 4H, $\mathrm{PCH}$ ), $3.88\left(\mathrm{~s}, 18 \mathrm{H}, \mathrm{CH}_{3}\right) .{ }^{13} \mathrm{C} \mathrm{NMR}\left(75 \mathrm{MHz}, \mathrm{CDCl}_{3}\right) \delta 195.3,164.7(\mathrm{~d}, J=$ 2.9), $136.5(\mathrm{~d}, J=11.5), 133.6(\mathrm{~d}, J=8.6), 131.7(\mathrm{~d}, J=5.3), 130.1(\mathrm{~d}, J=2.7), 115.9$ (d, $J=13.8), 109.1,107.9,56.0,31.6(\mathrm{~d}, J=48.7)$. LRMS $\left(\mathrm{ES}^{+}\right) m / z 911.46\left[(\mathrm{M}-\mathrm{H})^{+}\right]$, $455.97\left[\mathrm{M}^{2+}, 100 \%\right]$. ESI-HRMS $m / z 456.1683\left[\mathrm{M}^{2+}\right]\left(\mathrm{C}_{57} \mathrm{H}_{54} \mathrm{O}_{7} \mathrm{P}_{2}\right.$ requires 456.1667).

\section{4,4'-bis((tri(2-methoxyphenyl)phosphonio)methyl)diphenylmethane}

dibromide (43b). The reaction was carried out following the general procedure at $50{ }^{\circ} \mathrm{C}$ instead of $100{ }^{\circ} \mathrm{C}$ with tri-2-methoxyphenylphosphine and $\mathbf{1 b}$. The product was obtained as a white solid (259.7 mg, 93\%) following workup II procedure; mp 210-213 ${ }^{\circ} \mathrm{C}$ with previous softening (DMF/toluene); HPLC $>97 \%$ pure; ${ }^{1} \mathrm{H}$ NMR $(300 \mathrm{MHz}$, $\left.\mathrm{CDCl}_{3}\right) \delta 7.73(\mathrm{t}, J=7.9,6 \mathrm{H}, \mathrm{ArH}), 7.33-7.23(\mathrm{~m}, 8 \mathrm{H}, \mathrm{ArH}), 7.18-7.09(\mathrm{~m}, 10 \mathrm{H}$, $\operatorname{Ar} H), 6.88(\mathrm{~d}, J=8.0,4 \mathrm{H}, \operatorname{Ar} H), 6.79(\mathrm{~d}, J=8.0,4 \mathrm{H}, \operatorname{Ar} H), 4.58(\mathrm{~d}, J=15.9,4 \mathrm{H}$, $\mathbf{P C H}), 3.73\left(\mathrm{~s}, 2 \mathrm{H}, \mathrm{CH}_{2}\right), 3.63\left(\mathrm{~s}, 18 \mathrm{H}, \mathrm{CH}_{3}\right) .{ }^{13} \mathrm{C} \mathrm{NMR}\left(75 \mathrm{MHz}, \mathrm{CDCl}_{3}\right) \delta 161.4(\mathrm{~d}, J$ $=2.3), 140.5(\mathrm{dd}, J=3.4,1.2), 137.4(\mathrm{~d}, J=1.9), 135.2(\mathrm{~d}, J=8.2), 130.0(\mathrm{~d}, J=7.2)$, $129.1(\mathrm{~d}, J=2.3), 128.2(\mathrm{~d}, J=8.0), 122.1(\mathrm{~d}, J=12.8), 112.9(\mathrm{~d}, J=6.7), 105.7(\mathrm{~d}, J=$ 91.8), 56.5, 40.9, $30.7(\mathrm{~d}, J=52.7)$. LRMS $\left(\mathrm{ES}^{+}\right) \mathrm{m} / z 897.60\left[(\mathrm{M}-\mathrm{H})^{+}\right], 448.90\left[\mathrm{M}^{2+}\right.$, 100\%]. ESI-HRMS $m / z 449.1816\left[\mathrm{M}^{2+}\right]\left(\mathrm{C}_{57} \mathrm{H}_{56} \mathrm{O}_{6} \mathrm{P}_{2}\right.$ requires 449.1771).

\section{4,4'-bis((tri(2-methoxyphenyl)phosphonio)methyl)diphenylether}

dibromide (43c). The reaction was carried out following the general procedure at $50{ }^{\circ} \mathrm{C}$ instead of $100{ }^{\circ} \mathrm{C}$ with tri-2-methoxyphenylphosphine and 1c. The product was obtained 
as a white solid (231.3 mg, 85\%) following workup II procedure; mp $186-187{ }^{\circ} \mathrm{C}$ with previous softening (DMF/toluene); HPLC $>96 \%$ pure; ${ }^{1} \mathrm{H}$ NMR $\left(300 \mathrm{MHz}, \mathrm{CDCl}_{3}\right) \delta$ $7.74(\mathrm{t}, J=7.8,6 \mathrm{H}, \operatorname{Ar} H), 7.37-7.27(\mathrm{~m}, 6 \mathrm{H}, \operatorname{Ar} H), 7.19-7.11(\mathrm{~m}, 12 \mathrm{H}, \operatorname{Ar} H), 6.95$ $(\mathrm{dd}, J=17.0,15.0,4 \mathrm{H}, \mathrm{ArH}), 6.58(\mathrm{~d}, J=8.3,4 \mathrm{H}, \mathrm{Ar} H), 4.62\left(\mathrm{~d}, J=15.6,4 \mathrm{H}, \mathrm{PCH}{ }_{2}\right)$, $3.70\left(\mathrm{~s}, 18 \mathrm{H}, \mathrm{CH}_{3}\right) .{ }^{13} \mathrm{C} \mathrm{NMR}\left(75 \mathrm{MHz}, \mathrm{CDCl}_{3}\right) \delta 161.4(\mathrm{~d}, J=2.3), 156.4(\mathrm{~d}, J=3.5)$, $137.4(\mathrm{~d}, J=1.6), 135.2(\mathrm{~d}, J=8.2), 131.5(\mathrm{~d}, J=7.2), 125.4(\mathrm{~d}, J=7.8), 122.2(\mathrm{~d}, J=$ 12.8), $119.0(\mathrm{~d}, J=2.2), 112.9(\mathrm{~d}, J=6.8), 105.8(\mathrm{~d}, J=91.9), 56.5,30.3(\mathrm{~d}, J=52.6)$. LRMS $\left(\mathrm{ES}^{+}\right) \mathrm{m} / \mathrm{z} 899.63\left[(\mathrm{M}-\mathrm{H})^{+}\right], 449.88\left[\mathrm{M}^{2+}, 100 \%\right]$. ESI-HRMS $\mathrm{m} / \mathrm{z} 450.1699$ $\left[\mathrm{M}^{2+}\right]\left(\mathrm{C}_{56} \mathrm{H}_{54} \mathrm{O}_{7} \mathrm{P}_{2}\right.$ requires 450.1667).

4,4'-bis((tri-p-tolylphosphonio)methyl)benzophenone dibromide (45a). The reaction was carried out following the general procesude with tri-p-tolylphosphine and 1a. The product was obtained as a white solid (213.9 $\mathrm{mg}, 78 \%)$ following workup II procedure; mp $308.8{ }^{\circ} \mathrm{C}$ (decomp.); HPLC $>95 \%$ pure. ${ }^{1} \mathrm{H}$ NMR $\left(300 \mathrm{MHz}, \mathrm{CDCl}_{3}\right) \delta$ $7.61(\mathrm{dd}, J=12.4,8.2,12 \mathrm{H}, \operatorname{Ar} H), 7.40(\mathrm{dd}, J=8.1,3.1,12 \mathrm{H}, \operatorname{Ar} H), 7.35(\mathrm{~d}, J=8.2$, 4H, $\operatorname{Ar} H), 7.25-7.20(\mathrm{~m}, 4 \mathrm{H}, \mathrm{ArH}), 5.50(\mathrm{~d}, J=15.2,4 \mathrm{H}, \mathrm{PCH}), 2.45\left(\mathrm{~s}, 18 \mathrm{H}, \mathrm{CH}_{3}\right)$. ${ }^{13} \mathrm{C}$ NMR $\left(75 \mathrm{MHz}, \mathrm{CDCl}_{3}\right) \delta 195.4,146.3(\mathrm{~d}, J=3.0), 136.5(\mathrm{~d}, J=3.7), 134.5(\mathrm{~d}, J=$ $10.3), 133.2(\mathrm{~d}, J=8.6), 131.8(\mathrm{~d}, J=5.4), 131.0(\mathrm{~d}, J=13.1), 130.1(\mathrm{~d}, J=2.8), 114.6$ $(\mathrm{d}, J=88.7), 30.9(\mathrm{~d}, J=47.8), 22.0 . \mathrm{LRMS}\left(\mathrm{ES}^{+}\right) \mathrm{m} / \mathrm{z} 815.42\left[(\mathrm{M}-\mathrm{H})^{+}\right], 407.81\left[\mathrm{M}^{2+}\right.$, 100\%]. ESI-HRMS $m / z 408.1852\left[\mathrm{M}^{2+}\right]\left(\mathrm{C}_{57} \mathrm{H}_{54} \mathrm{OP}_{2}\right.$ requires 408.1819).

4,4'-bis((tri-p-tolylphosphonio)methyl)diphenylether dibromide (45c). The reaction was carried out following the general procedure with tri-p-tolylphosphine and 1c. The product was obtained as a white solid (149.3 $\mathrm{mg}, 57 \%)$ following workup II procedure and recrystallization from $\mathrm{EtOH} / \mathrm{Et}_{2} \mathrm{O} ; \mathrm{mp}>300{ }^{\circ} \mathrm{C}$ with previous softening $(\mathrm{DMF} / \mathrm{EtOH}) ; \mathrm{HPLC}>93 \%$ pure; ${ }^{1} \mathrm{H}$ NMR $\left(300 \mathrm{MHz}, \mathrm{CDCl}_{3}\right) \delta 7.54(\mathrm{dd}, J=12.2$, 8.2, 12H, ArH), $7.45-7.36(\mathrm{~m}, 12 \mathrm{H}, \operatorname{Ar} H), 7.08(\mathrm{dd}, J=8.4,2.2,4 \mathrm{H}, \operatorname{Ar} H), 6.66(\mathrm{~d}, J=$ 
8.4, 4H, $\mathrm{ArH}), 5.18(\mathrm{~d}, J=14.0,4 \mathrm{H}, \mathrm{PCH}), 2.44\left(\mathrm{~s}, 18 \mathrm{H}, \mathrm{CH}_{3}\right) .{ }^{13} \mathrm{C} \mathrm{NMR}(75 \mathrm{MHz}$, $\left.\mathrm{CDCl}_{3}\right) \delta 156.8(\mathrm{~d}, J=3.9), 146.3(\mathrm{~d}, J=3.2), 134.3(\mathrm{~d}, J=10.1), 133.1(\mathrm{~d}, J=5.4)$, $131.0(\mathrm{~d}, J=12.9), 122.6(\mathrm{~d}, J=8.5), 119.1(\mathrm{~d}, J=3.0), 114.7(\mathrm{~d}, J=88.4), 30.4(\mathrm{~d}, J=$ 48.2), 22.0. LRMS (ES $\left.{ }^{+}\right) \mathrm{m} / \mathrm{z} 803.59\left[(\mathrm{M}-\mathrm{H})^{+}\right], 401.93\left[\mathrm{M}^{2+}, 100 \%\right]$. ESI-HRMS m/z $402.1857\left[\mathrm{M}^{2+}\right]\left(\mathrm{C}_{56} \mathrm{H}_{54} \mathrm{OP}_{2}\right.$ requires 402.1819).

4,4'-bis((tri(p-tolyl)phosphonio)methyl)diphenylacetamide dibromide (45e). The reaction was carried out following the general procedure at $100{ }^{\circ} \mathrm{C}$ with tri-ptolylphosphine and 1e. The product was obtained as a highly hygroscopic white solid (72 mg, 53\%) following workup II procedure; HPLC $>96 \%$ pure; ${ }^{1} \mathrm{H}$ NMR $(300 \mathrm{MHz}$, $\left.\mathrm{CDCl}_{3}\right) \delta 7.63-7.47(\mathrm{~m}, 14 \mathrm{H}, \mathrm{Ar} H), 7.41(\mathrm{~m}, 10 \mathrm{H}, \mathrm{Ar} H), 7.15(\mathrm{~m}, 4 \mathrm{H}, \mathrm{Ar} H), 6.94(\mathrm{~d}, J$ $=7.4,4 \mathrm{H}, \operatorname{Ar} H), 5.25(\mathrm{~d}, J=15.8,4 \mathrm{H}, \mathrm{PCH}), 2.45\left(\mathrm{~s}, 18 \mathrm{H}, \mathrm{CH}_{3}\right), 1.94(\mathrm{~s}, 3 \mathrm{H}$, $\left.\mathrm{COCH}_{3}\right) .{ }^{13} \mathrm{C}$ NMR $\left(75 \mathrm{MHz}, \mathrm{CDCl}_{3}\right) \delta 170.4,146.5(\mathrm{~d}, J=2.7), 142.7(\mathrm{~d}, J=3.0)$, $134.3(\mathrm{~d}, J=10.2), 133.4-132.1(\mathrm{br}), 131.1(\mathrm{~d}, J=12.9), 114.5(\mathrm{~d}, J=88.6), 30.8(\mathrm{~d}, J$ $=48.2), 24.0,22.0$. LRMS $\left(\mathrm{ES}^{+}\right) \mathrm{m} / z$ 844.61 $\left[(\mathrm{M}-\mathrm{H})^{+}\right], 422.58\left[\mathrm{M}^{2+}, 100 \%\right]$. ESIHRMS $m / z 422.6984\left[\mathrm{M}^{2+}\right]\left(\mathrm{C}_{58} \mathrm{H}_{57} \mathrm{NOP}_{2}\right.$ requires 422.6952$)$.

4,4'-bis((tri-2-thienylphosphonio)methyl)benzophenone dibromide (47a). The reaction was carried out following the general procedure with tri-2thienylphosphine and $\mathbf{1 a}(110 \mathrm{mg}, 0.3 \mathrm{mmol})$ for $18 \mathrm{~h}$. The product was obtained as an off-white solid after workup II and recrystallization from EtOH (200 mg, 72\%); HPLC> 95\% pure. ${ }^{1} \mathrm{H}$ NMR $\left(300 \mathrm{MHz}, \mathrm{CDCl}_{3}\right) \delta 8.12(\mathrm{~m}, 12 \mathrm{H}, \mathrm{Ar} H), 7.41(\mathrm{dt}, J=6.8,6.2$, $10 \mathrm{H}, \mathrm{Ar} H), 7.24(\mathrm{dd}, J=8.2,2.6,4 \mathrm{H}, \mathrm{Ar} H), 5.26(\mathrm{~d}, J=15.4,4 \mathrm{H}, \mathrm{PCH}) .{ }^{13} \mathrm{C} \mathrm{NMR}(75$ $\left.\mathrm{MHz}, \mathrm{CDCl}_{3}\right) \delta 195.3,143.1(\mathrm{~d}, J=11.8), 140.3(\mathrm{~d}, J=5.4), 137.1(\mathrm{~d}, J=4.3), 132.0$ $(\mathrm{d}, J=9.5), 131.5(\mathrm{~d}, J=6.1), 131.0(\mathrm{~d}, J=16.1), 130.4(\mathrm{~d}, J=3.6), 117.1(\mathrm{~d}, J=$ 109.4), $36.0(\mathrm{~d}, J=53.4)$. LRMS $\left(\mathrm{ES}^{+}\right) \mathrm{m} / z$ 767.1 $\left[(\mathrm{M}-\mathrm{H})^{+}\right], 383.74\left[\mathrm{M}^{2+}, 100 \%\right]$. ESIHRMS $m / z 384.0085\left[\mathrm{M}^{2+}\right]\left(\mathrm{C}_{39} \mathrm{H}_{30} \mathrm{OS}_{6} \mathrm{P}_{2}\right.$ requires 384.0043). 


\section{4,4'-bis((tri(1-naphthyl)phosphonio)methyl)diphenylmethane}

dibromide (53b). The reaction was carried out following the general procedure with tri1-naphthylphosphine and 1b. The product was obtained as a white solid (229.2 $\mathrm{mg}$, $71 \%$ ) following workup I procedure and recrystallization from $\mathrm{EtOH} / \mathrm{Et}_{2} \mathrm{O} ; \mathrm{mp} 230{ }^{\circ} \mathrm{C}$ (decomp); HPLC > 95\% pure; ${ }^{1} \mathrm{H}$ NMR $\left(300 \mathrm{MHz}, \mathrm{CDCl}_{3}\right) \delta 8.56(\mathrm{~d}, J=11.5,6 \mathrm{H}$, $\operatorname{Ar} H), 8.23(\mathrm{~d}, J=7.5,6 \mathrm{H}, \operatorname{Ar} H), 7.89(\mathrm{dd}, J=26.8,8.0,12 \mathrm{H}, \operatorname{Ar} H), 7.68(\mathrm{br} \mathrm{s}, 6 \mathrm{H}$, $\operatorname{Ar} H), 7.41(\mathrm{dt}, J=14.7,7.1,12 \mathrm{H}, \operatorname{Ar} H), 6.72(\mathrm{~d}, J=7.0,4 \mathrm{H}, \operatorname{Ar} H), 6.25(\mathrm{~d}, J=7.0$, 4H, $\mathrm{ArH}), 5.66(\mathrm{~d}, J=11.3,4 \mathrm{H}, \mathrm{PCH}), 3.30\left(\mathrm{~s}, 2 \mathrm{H}, \mathrm{CH}_{2}\right) .{ }^{13} \mathrm{C} \mathrm{NMR}\left(75 \mathrm{MHz}, \mathrm{CDCl}_{3}\right)$ $\delta 140.5(\mathrm{~d}, J=2.6), 138.5(\mathrm{~d}, J=10.8), 137.1(\mathrm{~d}, J=2.8), 134.3(\mathrm{~d}, J=9.6), 132.6(\mathrm{~d}, J$ $=8.4), 130.7(\mathrm{~d}, J=6.7), 130.5,129.0,128.8(\mathrm{~d}, J=2.1), 127.6,126.7(\mathrm{~d}, J=7.5)$, $125.9-125.6(\mathrm{~m}), 114.6(\mathrm{~d}, J=80.9), 40.6,33.3(\mathrm{~d}, J=48.1)$. LRMS $\left(\mathrm{ES}^{+}\right) \mathrm{m} / \mathrm{z}$ $1017.77\left[(\mathrm{M}-\mathrm{H})^{+}\right], 508.96\left[\mathrm{M}^{2+}, 100 \%\right]$. ESI-HRMS m/z 509.1968 $\left[\mathrm{M}^{2+}\right]\left(\mathrm{C}_{75} \mathrm{H}_{56} \mathrm{P}_{2}\right.$ requires 509.1923).

4,4'-bis((tri-p-tolylphosphonio)ethyl)diphenylethane dichloride (64). The reaction was carried out following the general procedure with tri-p-tolylphosphine and 16f for 15 days. The reaction was evaporated to dryness forming a yellowish oil which was purified by semi-preparative HPLC-MS. IThe following HPLC conditions were used: column temperature $=25{ }^{\circ} \mathrm{C}$, gradient time $=10 \mathrm{~min}, \mathrm{CH}_{3} \mathrm{CN} / \mathrm{H}_{2} \mathrm{O}$ $(30: 70 \rightarrow 100: 0)\left(\mathrm{HCO}_{2} \mathrm{H} 0.1 \%\right)$, flow rate $=0.24 \mathrm{~mL} / \mathrm{min}, \mathrm{UV}$ detection: diode array $(\lambda$ $=230 \mathrm{~nm}) . R_{\mathrm{t}}=6.38 \mathrm{~min}$. The fractions containing the pure product were combined and lyophilized to give a white hygroscopic solid (12.6 mg, 5\%). HPLC > 99\% pure;. ${ }^{1} \mathrm{H}$ $\operatorname{NMR}\left(300 \mathrm{MHz}, \mathrm{CDCl}_{3}\right) \delta 7.62(\mathrm{dd}, J=12.3,8.1,12 \mathrm{H}, \mathrm{Ar} H), 7.54-7.41(\mathrm{~m}, 12 \mathrm{H}$, $\operatorname{ArH}), 7.09(\mathrm{~d}, J=7.8,4 \mathrm{H}, \operatorname{Ar} H), 6.95(\mathrm{~d}, J=7.8,4 \mathrm{H}, \operatorname{Ar} H), 3.79-3.58(\mathrm{~m}, 4 \mathrm{H}$, PCH $\mathrm{PH}_{2}, 2.99-2.84\left(\mathrm{~m}, 4 \mathrm{H}, \mathrm{PhCH}_{2} \mathrm{CH}_{2} \mathrm{P}\right), 2.81\left(\mathrm{~s}, 4 \mathrm{H}, \mathrm{CH}_{2} \mathrm{CH}_{2}\right), 2.48\left(\mathrm{~s}, 18 \mathrm{H}, \mathrm{CH}_{3}\right)$. ${ }^{13} \mathrm{C}$ NMR $\left(75 \mathrm{MHz}, \mathrm{CDCl}_{3}\right) \delta 146.8,142.6(\mathrm{~d}, J=2.5), 136.7,133.5(\mathrm{dd}, J=10.1,2.4)$, 
$132.2(\mathrm{~d}, J=10.4), 129.3(\mathrm{~d}, J=12.6), 128.7,114.6(\mathrm{dd}, J=88.1,2.6), 37.6,35.0,28.1$ $(\mathrm{d}, J=1.4), 22.0$. LRMS $\left(\mathrm{ES}^{+}\right) \mathrm{m} / \mathrm{z}=843.39[(\mathrm{M}-\mathrm{H})]^{+}, 422.00\left[\mathrm{M}^{2+}, 100 \%\right]$. ESIHRMS $m / z 422.2181\left[\mathrm{M}^{2+}\right]\left(\mathrm{C}_{60} \mathrm{H}_{62} \mathrm{P}_{2}\right.$ requires 422.2158).

4,4'-bis((tri-p-tolylphosphonio)ethyl)diphenylpropane dichloride (65). The reaction was carried out following the general procedure with tri-p-tolylphosphine and $\mathbf{1 7 g}$ for 19 days. The reaction was evaporated to dryness forming a yellowish oil which was purified by semi-preparative HPLC-MS eluting with $\mathrm{H}_{2} \mathrm{O} / \mathrm{CH}_{3} \mathrm{CN}$. The following HPLC conditions were used: column temperature $=25{ }^{\circ} \mathrm{C}$, gradient time $=15 \mathrm{~min}$, $\mathrm{CH}_{3} \mathrm{CN} / \mathrm{H}_{2} \mathrm{O}(30: 70 \rightarrow 70: 30)\left(\mathrm{HCO}_{2} \mathrm{H} 0.1 \%\right)$, flow rate $=0.24 \mathrm{~mL} / \mathrm{min}$, UV detection: diode array $(\lambda=240 \mathrm{~nm}) . R_{\mathrm{t}}=10.32 \mathrm{~min}$. The fractions containing the pure product were combined and lyophilized to give a white hygroscopic solid (17.3 mg, 17\%). HPLC $>99 \%$ pure. ${ }^{1} \mathrm{H}$ NMR $\left(300 \mathrm{MHz}, \mathrm{CDCl}_{3}\right) \delta 7.72-7.55(\mathrm{~m}, 12 \mathrm{H}, \mathrm{ArH}), 7.53-$ $7.43(\mathrm{~m}, 12 \mathrm{H}, \operatorname{Ar} H), 7.14(\mathrm{~d}, J=7.4,4 \mathrm{H}, \operatorname{Ar} H), 7.04$ (d, $J=7.4,4 \mathrm{H}, \operatorname{Ar} H), 3.78$ (br m, 4H, $\mathrm{PCH}$ ), 2.95 (br m, 4H, $\left.\mathrm{CH}_{2}\right), 2.60-2.41\left(\mathrm{~m}, 22 \mathrm{H}, \mathrm{CH}_{2}\right.$ and $\left.\mathrm{CH}_{3}\right), 1.86(\mathrm{dt}, \mathrm{J}=14.3$, 7.4, 2H, $\left.\mathrm{CH}_{2} \mathrm{CH}_{2} \mathrm{CH}_{2}\right) .{ }^{13} \mathrm{C} \mathrm{NMR}\left(75 \mathrm{MHz}, \mathrm{CDCl}_{3}\right) \delta 146.4(\mathrm{dd}, J=5.1,3.0), 142.3(\mathrm{~d}$, $J=2.8), 135.5(\mathrm{~d}, J=24.9), 133.6(\mathrm{dd}, J=10.4,2.6), 132.2(\mathrm{~d}, J=10.3), 129.3(\mathrm{~d}, J=$ 12.5), $128.6(\mathrm{~d}, J=7.2), 115.1(\mathrm{dd}, J=88.4,7.2), 34.6,33.0,28.1(\mathrm{~d}, J=3.1), 24.8(\mathrm{~d}, J$ $=50.1), 22.0$. LRMS $\left(\mathrm{ES}^{+}\right) \mathrm{m} / \mathrm{z}=857.88[(\mathrm{M}-\mathrm{H})]^{+}, 429.21\left[\mathrm{M}^{2+}, 100 \%\right]$. ESI-HRMS $m / z 429.2277\left[\mathrm{M}^{2+}\right]\left(\mathrm{C}_{61} \mathrm{H}_{64} \mathrm{P}_{2}\right.$ requires 429.2236).

2. General procedure for the synthesis of the monophosphonium salts. The appropriate halogenated precursor $(100 \mathrm{mg}, \sim 0.36 \mathrm{mmol})$ was added to a Kimax tube and dissolved in anhydrous DMF (3 mL) under argon atmosphere. The phosphine was then added ( 0.72 mmol, 2 equiv.) and the reaction mixture was stirred at $100{ }^{\circ} \mathrm{C}$ for 20 h. Next, the reaction mixture was transferred to a flask and DMF was evaporated under 
vacuum. Subsequently, toluene $(10-20 \mathrm{~mL})$ was added to precipitate the product and the flask was stored in the fridge overnight. The supernatant was removed and the precipitate was rinsed with toluene. $\mathrm{Et}_{2} \mathrm{O}(10 \mathrm{~mL})$ was added and the precipitate was triturated with a spatula. The solid was collected, rinsed with $\mathrm{Et}_{2} \mathrm{O}$ and dried under vacuum.

(4-benzoylbenzyl)tri-p-tolylphosphonium bromide (56a). The reaction was carried out following the general procedure with $\mathbf{2 a}$ and tri-p-tolylphosphine. The product was obtained as a white solid following workup II procedure (117.6 mg, 56\%). HPLC $>95 \%$ pure; mp $233{ }^{\circ} \mathrm{C} .{ }^{1} \mathrm{H}$ NMR $\left(300 \mathrm{MHz}, \mathrm{CDCl}_{3}\right) \delta 7.68(\mathrm{dd}, J=3.0,6.5$, 2H, $\operatorname{Ar} H), 7.62-7.50(\mathrm{~m}, 9 \mathrm{H}, \operatorname{Ar} H), 7.47-7.32(\mathrm{~m}, 8 \mathrm{H}, \operatorname{Ar} H), 7.28(\mathrm{dd}, J=2.3,8.0,2 \mathrm{H}$, $\mathrm{ArH}), 5.44(\mathrm{~d}, J=15.0,2 \mathrm{H}, \mathrm{PCH}), 2.42\left(\mathrm{~s}, 9 \mathrm{H}, \mathrm{CH}_{3}\right) .{ }^{13} \mathrm{C} \mathrm{NMR}\left(75 \mathrm{MHz}, \mathrm{CDCl}_{3}\right) \delta$ $196.3,146.4(\mathrm{~d}, J=3.0), 137.2(\mathrm{~d}, J=3.8), 137.1,134.3(\mathrm{~d}, J=10.3), 132.8,132.7$, $131.7(\mathrm{~d}, J=5.4), 130.9(\mathrm{~d}, J=13.0), 130.2(\mathrm{~d}, J=3.1), 130.1,128.4,114.4(\mathrm{~d}, J=$ 88.7), $31.1(\mathrm{~d}, J=48.3), 21.9$. LRMS $\left(\mathrm{ES}^{+}\right) \mathrm{m} / \mathrm{z}=499.44\left[\mathrm{M}^{+}\right]$. ESI-HRMS $\mathrm{m} / \mathrm{z}$ $499.2203\left[\mathrm{M}^{+}\right]\left(\mathrm{C}_{35} \mathrm{H}_{32} \mathrm{OP}\right.$ requires 499.2185).

(4-phenoxybenzyl)tri-p-tolylphosphonium bromide (56c). The reaction was carried out following the general procedure with $\mathbf{2 c}$ and tri-p-tolylphosphine. The product was obtained as a white solid following workup II procedure (94.7 mg, 87\%). HPLC $>95 \%$ pure; mp $192{ }^{\circ} \mathrm{C} .{ }^{1} \mathrm{H}$ NMR $\left(300 \mathrm{MHz}, \mathrm{CDCl}_{3}\right) \delta 7.56(\mathrm{dd}, J=7.8,12.2$, 6H, $\operatorname{Ar} H), 7.47-7.00(\mathrm{~m}, 11 \mathrm{H}, \operatorname{Ar} H), 6.93(\mathrm{~m}, 2 \mathrm{H}, \operatorname{Ar} H), 6.76(\mathrm{~d}, J=8.1,2 \mathrm{H}, \operatorname{Ar} H)$, $5.20(\mathrm{~d}, J=13.9,2 \mathrm{H}, \mathrm{PCH}), 2.45\left(\mathrm{~s}, 9 \mathrm{H}, \mathrm{CH}_{3}\right) .{ }^{13} \mathrm{C} \mathrm{NMR}\left(75 \mathrm{MHz}, \mathrm{CDCl}_{3}\right) \delta 157.5(\mathrm{~d}$, $J=3.8), 156.7,146.3(\mathrm{~d}, J=2.8), 134.4(\mathrm{~d}, J=10.1), 133.1(\mathrm{~d}, J=5.3), 131.0(\mathrm{~d}, J=$ 12.9), $129.1,123.8,122.0(\mathrm{~d}, J=8.5), 119.1,119.0(\mathrm{~d}, J=2.9), 114.8(\mathrm{~d}, J=88.3), 30.6$ ( $\mathrm{d}, J=48.4), 22.0 . \mathrm{LRMS}\left(\mathrm{ES}^{+}\right) \mathrm{m} / \mathrm{z}=487.40\left[\mathrm{M}^{+}\right]$. ESI-HRMS $m / z 487.2206\left[\mathrm{M}^{+}\right]$ $\left(\mathrm{C}_{34} \mathrm{H}_{32} \mathrm{OP}\right.$ requires 487.2185$)$. 
(4-phenoxybenzyl)tri-m-tolylphosphonium bromide (57c). The reaction was carried out following the general procedure with $\mathbf{2 c}$ and tri-m-tolylphosphine. The product was obtained as a white solid following workup II procedure (65.2 $\mathrm{mg}, 60 \%)$. mp $228.9^{\circ} \mathrm{C} .{ }^{1} \mathrm{H}$ NMR $\left(300 \mathrm{MHz}, \mathrm{CDCl}_{3}\right) \delta 7.58-7.46(\mathrm{~m}, 11 \mathrm{H}, \mathrm{Ar} H), 7.29(\mathrm{dd}, J=$ 14.5, 6.2, 3H, ArH), $7.04(\mathrm{dd}, J=8.7,2.4,3 \mathrm{H}, \operatorname{Ar} H), 6.93(\mathrm{~d}, J=7.6,2 \mathrm{H}, \operatorname{Ar} H), 6.75$ $(\mathrm{d}, J=8.2,2 \mathrm{H}, \mathrm{Ar} H), 5.29(\mathrm{~d}, J=13.8,2 \mathrm{H}, \mathrm{PCH} 2), 2.41\left(\mathrm{~s}, 9 \mathrm{H}, \mathrm{CH}_{3}\right) .{ }^{13} \mathrm{C} \mathrm{NMR}(75$ $\left.\mathrm{MHz}, \mathrm{CDCl}_{3}\right) \delta 157.7,156.6,140.6(\mathrm{~d}, J=12.4), 135.9(\mathrm{~d}, J=2.9), 134.6(\mathrm{~d}, J=9.7)$, $133.1(\mathrm{~d}, J=5.4), 131.6(\mathrm{~d}, J=9.7), 130.4(\mathrm{~d}, J=13.2), 128.9,123.9,121.9(\mathrm{~d}, J=$ 8.6), 119.3, $\left.118.8(\mathrm{~d}, J=3.1), 117.9(\mathrm{~d}, J=84.8), 30.4(\mathrm{~d}, J=47.1), 21.6 . \mathrm{LRMS}_{(\mathrm{ES}}{ }^{+}\right)$ $\mathrm{m} / \mathrm{z}=487.40[\mathrm{M}]^{+}$. Anal. $\left(\mathrm{C}_{32} \mathrm{H}_{50} \mathrm{BrOP}\right)$ Calc: $\mathrm{C}, 71.96 ; \mathrm{H}, 5.68 ; \mathrm{Br}, 14.08$. Found: $\mathrm{C}$, 72.18; H, 5.71; Br, 13.93.

(4-benzoylbenzyl)tri-n-hexylphosphonium bromide (62a). The reaction was carried out following the general procedure with 2a and tri-n-hexylphosphine. The product was obtained as a white solid following workup II procedure (84.3 $\mathrm{mg}, 52 \%)$. HPLC > 95\% pure; mp $109.1{ }^{\circ} \mathrm{C} .{ }^{1} \mathrm{H}$ NMR $\left(300 \mathrm{MHz}, \mathrm{CDCl}_{3}\right) \delta 7.81-7.64(\mathrm{~m}, 6 \mathrm{H}$, $\operatorname{Ar} H), 7.63-7.55(\mathrm{~m}, 1 \mathrm{H}, \operatorname{Ar} H), 7.47(\mathrm{t}, J=7.5,2 \mathrm{H}, \operatorname{Ar} H), 4.58(\mathrm{~d}, J=15.9,2 \mathrm{H}$, $\left.\mathrm{PhCH}_{2}\right), 2.51-2.29\left(\mathrm{~m}, 6 \mathrm{H}, \mathrm{PCH}_{2} \mathrm{CH}_{2}\right), 1.42\left(\mathrm{~s}, 12 \mathrm{H}, \mathrm{CH}_{2}\right), 1.33-1.12\left(\mathrm{~m}, 12 \mathrm{H}, \mathrm{CH}_{2}\right)$, $0.84\left(\mathrm{t}, J=6.7,9 \mathrm{H}, \mathrm{CH}_{3}\right) .{ }^{13} \mathrm{C} \mathrm{NMR}\left(75 \mathrm{MHz}, \mathrm{CDCl}_{3}\right) \delta 195.9,137.6(\mathrm{~d}, J=3.6)$, $137.0,133.7(\mathrm{~d}, J=8.9), 133.0,131.0(\mathrm{~d}, J=2.9), 130.4(\mathrm{~d}, J=4.9), 129.3(\mathrm{~d}, J=$ 115.5), 31.1, $30.6(\mathrm{~d}, J=14.8), 22.4,22.0(\mathrm{~d}, J=4.8), 19.2(\mathrm{~d}, J=46.1), 14.0$. LRMS $\left(\mathrm{ES}^{+}\right) \mathrm{m} / \mathrm{z}=482.36[(\mathrm{M}+\mathrm{H})]^{+}$. Anal. $\left(\mathrm{C}_{32} \mathrm{H}_{50} \mathrm{BrOP}\right)$ Calc: C, 68.56; H, 8.81; Br, 14.25 . Found: C, 68.59; H, 8.90; Br, 14.41.

\section{Biology.}


In vitro antitrypanosomal activity. The in vitro trypanocidal and cytotoxic activities were determined using the Alamar blue assay. ${ }^{27}$ Detailed experimental protocols for these assays with T. b. rhodesiense STIB900 and L6-cells have been reported before. ${ }^{28} \mathrm{~A}$ slight modification of this protocol was used for the assays with wild type and resistant $T$. b. brucei strains, as described. ${ }^{29}$ The TbAT1-KO strain is derived from the wild type strain T. b. brucei Lister 427 (s427) by deletion of the TbAT1 gene. ${ }^{18}$ The B48 strain is a mutant derived from TbAT1-KO by in vitro selection to high levels of pentamidine, and does not express a functional High Affinity Pentamidine Transporter (HAPT1). ${ }^{8}$ Monitoring of trypanosome cellular integrity with propidium iodide was performed exactly as described previously. ${ }^{25}$

Pentamidine transport assays. Uptake assays used $\left[{ }^{3} \mathrm{H}\right]$-pentamidine (Amersham) at either $30 \mathrm{nM}$ final concentration for the assessment of HAPT1-mediated transport, or at $1 \mu \mathrm{M}$ for the assessment of LAPT1-mediated transport, exactly as described. ${ }^{20,30}$ Briefly, trypanosomes were grown in HMI-9 with 10\% Fetal Bovine Serum (FBS) until late-log phase, harvested and washed into assay buffer (33 mM HEPES, $98 \mathrm{mM} \mathrm{NaCl}$, 4.6 mM KCl, $0.55 \mathrm{mM} \mathrm{CaCl}_{2}, 0.07 \mathrm{mM} \mathrm{MgSO}_{4}, 5.8 \mathrm{mM} \mathrm{NaH}_{2} \mathrm{PO}_{4}, 0.3 \mathrm{mM} \mathrm{MgCl}_{2}, 23$ $\mathrm{mM} \mathrm{NaHCO} 3,14 \mathrm{mM}$ glucose, $\mathrm{pH}$ 7.3) prior to use in the assay. Cells were incubated with radiolabel for $60 \mathrm{~s}$ or $120 \mathrm{~s}$ for HAPT1 and LAPT1 assays, respectively. Incubations were stopped by the addition of $2 \mathrm{mM}$ ice-cold unlabeled pentamidine and immediate centrifugation through oil. Quantification was through scintillation counting and curves were fitted to sigmoid curves using the Prism 5.0 software package. All experiments were performed in triplicate and on at least three fully independent occasions. 
CoMFA Models. The set of compounds (78 in total) were converted to 3D structures using LigPrep ${ }^{31}$ considering $\mathrm{pH} 7.0$ for the protonation state. Nevertheless, only one compound within the set, 49a, showed an additional protonation at the fixed $\mathrm{pH}$ for the pyridine moiety. Both states were modelled and it turned out that the protonated state was more consistent with the modelled data. We removed all counter ions, which is why compounds 45a, 45f, 45g, 64 and 65 were not included in the modelling step. Atomic charges used for the CoMFA models were calculated at the $\mathrm{AM} 1^{32}$ semiempirical level using Gaussian $03 .^{33}$

The whole set was divided into two subsets: training compounds and test compounds in order to properly validate the models. It was considered as important to have a good balance between active and inactive compounds within the test set for the sake of keeping a predictive model. With this goal in mind the whole set was divided into active, less active and inactive compounds, doing the random selection upon these three subsets trying to keep a similar number of compounds among the three subsets. However, during the modelling step the activities $\left(\mathrm{EC}_{50}\right)$ of some compounds were still to be determined, so these compounds were included in the test set.

The molecular alignment was built with the flexible molecular overlay method $(50 \%$ steric, $50 \%$ electrostatic) as included in Discovery Studio, Accelerys, ${ }^{34}$ using the selected conformations of the more active compound in the training set as reference. Erroneously fitted compounds were re-aligned manually defining pairs of atoms ensuring a maximum molecular surface overlay. The resulting conformations on the whole set were checked to verify that no unusual energy penalty is paid for the resulting conformations. 
Four different alignments were used within this work based on the four more stable conformations, whilst keeping significantly different Root Mean Square Deviations (RMSD), found for the more active compound. Here we only present the results on one alignment, the one corresponding to the lowest energy conformations, given that it yields the better results. In any case no remarkable differences were found for the four calculated molecular overlays. CoMFA descriptors calculation (standard CoMFA, HB and index), PLS model fitting, region focusing and models validations were conducted in the standard manner described within the Sybyl suite ${ }^{35}$ and we found that standard CoMFA descriptors were perfectly able to model the proposed end point. Energetic cut offs for CoMFA descriptors were set to $30 \mathrm{kcal} \mathrm{mol}^{-1}$, which was more suitable for $T$. b. brucei than other calculated values.

Acknowledgements. This work was supported by grants from the Spanish Ministerio de Ciencia e Innovación (Grants SAF2006-04698, SAF2009-10399), and the CSIC (bilateral project grant 2008GB0021) to C.D., the UNDP/World Bank/WHO Special Program for Research and Training in Tropical Diseases (M.K.). C.R. was recipient of a PhD fellowship for the government of Panama (SENACYT grant BIDP2008-030). EFP was recipient of grants from the CSIC (JAE INTRO 08/09). VH was recipient of JAE-TEC contract from the CSIC. HdK acknowledges support from the Royal Society (International Joint Project JP0872898), the UK Commonwealth Office (Studentship to AAE) and the government of Saudi Arabia (studentship to AA, Aljouf University, Saudi Arabia). A.C. wants to thank the JAE-DOC program for his contract. 
Supporting Information Available: Synthesis and characterization of the new phosphonium salt derivatives 19c, 23b-e, 25c, 28b-c, 33b-c, 34b-c, 35a, 38b, 39a, 40a, 42a, 43a, 44a-c, 45a, 45b, 45d, 45f, 45g, 46b-c, 50a, 51b-c, 52b-c, 53a, 53c, 57a-61a, 63a, and the linkers 2a, 3a, 10f, 11g, 12f, 13g, 16f and 17g. QSAR results table and molecular overlay. Effect of compounds 25b, 25c, 26a, 35a, 36a, 38a, 43a, 43c, 45d, 45e, $47 \mathbf{a}$, and 55 on parasite viability as determined with the propidium iodide assay. This material is available free of charge via the internet at http://pubs.acs.org.

\section{References}

1. WHO. Working to overcome the global impact of neglected tropical diseases: first WHO report on neglected tropical diseases; World Health Organization: Geneva, 2010.

2. Delespaux, V.; de Koning, H. P. Drugs and drug resistance in African trypanosomiasis. Drug Resist. Updates 2007, 10, 30-50.

3. Rodgers, J. Human African trypanosomiasis, chemotherapy and CNS disease. $J$. Neuroimmunol. 2009, 211, 16-22.

4. Kinnamon, K. E.; Steck, E. A.; Rane, D. S. A new chemical series active against African trypanosomes: benzyltriphenylphosphonium salts. J. Med. Chem. 1979, 22, $452-455$.

5. Luque-Ortega, J. R.; Reuther, P.; Rivas, L.; Dardonville, C. New BenzophenoneDerived Bisphosphonium Salts as Leishmanicidal Leads Targeting Mitochondria through Inhibition of Respiratory Complex II. J. Med. Chem. 2010, 53, 1788-1798.

6. Dardonville, C.; Brun, R. Bisguanidine, bis(2-aminoimidazoline), and polyamine derivatives as potent and selective chemotherapeutic agents against Trypanosoma brucei rhodesiense. Synthesis and in vitro evaluation. J. Med. Chem. 2004, 47, 22962307. 
7. de Koning, H. P. Ever-increasing complexities of diamidine and arsenical crossresistance in African trypanosomes. Trends Parasitol. 2008, 24, 345-349.

8. Bridges, D. J.; Gould, M. K.; Nerima, B.; Ma?ser, P.; Burchmore, R. J. S.; De Koning, H. P. Loss of the high-affinity pentamidine transporter is responsible for high levels of cross-resistance between arsenical and diamidine drugs in african trypanosomes. Mol. Pharmacol. 2007, 71, 1098-1108.

9. Lüscher, A.; de Koning, H. P.; MÃđser, P. Chemotherapeutic strategies against Trypanosoma brucei: Drug targets vs. drug targeting. Curr. Pharm. Design 2007, 13, $555-567$.

10. de Koning, H. P.; Anderson, L. F.; Stewart, M.; Burchmore, R. J.; Wallace, L. J.; Barrett, M. P. The trypanocide diminazene aceturate is accumulated predominantly through the TbAT1 purine transporter: additional insights on diamidine resistance in african trypanosomes. Antimicrob Agents Chemother 2004, 48, 1515-1519.

11. Golden, J. H. Poly-p-xylylene and related compounds. J. Chem. Soc. 1961, 1604-1610.

12. Jung, H. K.; Lee, J. K.; Kang, M. S.; Kim, S. W.; Kim, J. J.; Park, S. Y. Synthesis and properties of poly(p-phenylenevinylene-co-sulfonylene) for a blue lightemitting diode. Polymer Bull. 1999, 43, 13-20.

13. Wakselman, M.; Domé, M. Alkylations en milieu aqueux par les halogénures benzyliques aminés ou amidés. 1. Synthèse de réactifs mono- et bialkylants. Bull. Chem. Soc. Fr. 1975, 571-575.

14. Boyer, B.; Keramane, E. M.; Arpin, S.; Montéro, J.-L.; Roque, J.-P. BiX3 as an efficient and selective reagent for the halogen exchange reaction. Tetrahedron 1999, 55, 1971-1976. 
15. Grützmacher, H. F.; Neumann, E.; Ebmeyer, F.; Albrecht, K.; Schelenz, P. [3.2]Paracyclophane-10-enes and [3.2.3.2]Paracyclophane-10,27-dienes: A convenient synthesis by the McMurry reaction and dynamic stereochemistry. Chem. Ber. 1989, 122, 2291-2297.

16. Berg, M.; Kohl, L.; Van der Veken, P.; Joossens, J.; Al-Salabi, M. I.; Castagna, V.; Giannese, F.; Cos, P.; Versees, W.; Steyaert, J.; Grellier, P.; Haemers, A.; Degano, M.; Maes, L.; de Koning, H. P.; Augustyns, K. Evaluation of nucleoside hydrolase inhibitors for treatment of African trypanosomiasis. Antimicrob. Agents Chemother. 2010, 54, 1900-1908.

17. Gould, M. K.; Vu, X. L.; Seebeck, T.; de Koning, H. P. Propidium iodide-based methods for monitoring drug action in the kinetoplastidae: Comparison with the Alamar Blue assay. Anal. Biochem. 2008, 382, 87-93.

18. Matovu, E.; Stewart, M. L.; Geiser, F.; Brun, R.; MÃøser, P.; Wallace, L. J. M.; Burchmore, R. J.; Enyaru, J. C. K.; Barrett, M. P.; Kaminsky, R.; Seebeck, T.; De Koning, H. P. Mechanisms of arsenical and diamidine uptake and resistance in Trypanosoma brucei. Eukaryot. Cell 2003, 2, 1003-1008.

19. Ross, M. F.; Da Ros, T.; Blaikie, F. H.; Prime, T. A.; Porteous, C. M.; Severina, I. I.; Skulachev, V. P.; Kjaergaard, H. G.; Smith, R. A. J.; Murphy, M. P. Accumulation of lipophilic dications by mitochondria and cells. Biochem. J. 2006, 400, 199-208.

20. de Koning, H. P. Uptake of pentamidine in Trypanosoma brucei brucei is mediated by three distinct transporters: Implications for cross-resistance with arsenicals. Mol. Pharmacol. 2001, 59, 586-592.

21. Bush, B. L.; Nachbar, R. B. Sample-distance Partial Least Squares: PLS optimized for many variables, with application to CoMFA. J. Computer-Aided Molec. Design 1993, 587-619. 
22. Golbraikh, A.; Tropsha, A. Beware of q2! J. Mol. Graphics. Model. 2002, 20, 269-276.

23. Ross, M.; Kelso, G.; Blaikie, F.; James, A.; Cochemé, H.; Filipovska, A.; Da Ros, T.; Hurd, T.; Smith, R.; Murphy, M. Lipophilic triphenylphosphonium cations as tools in mitochondrial bioenergetics and free radical biology. Biochemistry (Moscow) 2005, 70, 222-230.

24. Teka, I. A.; Kazibwe, A. J. N.; El-Sabbagh, N.; Al-Salabi, M. I.; Ward, C. P.; Eze, A. A.; Munday, J. C.; Mäser, P.; Matovu, E.; Barrett, M. P.; De Koning, H. P. The diamidine diminazene aceturate is a substrate for the high-affinity pentamidine transporter: Implications for the development of high resistance levels in trypanosomes. Mol. Pharmacol. 2011, 80, 110-116.

25. Ibrahim, H. M. S.; Al-Salabi, M. I.; Sabbagh, N. E.; Quashie, N. B.; Alkhaldi, A. A. M.; Escale, R.; Smith, T. K.; Vial, H. J.; de Koning, H. P. Symmetrical cholinederived dications display strong anti-kinetoplastid activity. J. Antimicrob. Chemother. 2011, 66, 111-125.

26. Ward, C. P.; Wong, P. E.; Burchmore, R. J.; De Koning, H. P.; Barrett, M. P. Trypanocidal furamidine analogues: Influence of pyridine nitrogens on trypanocidal activity, transport kinetics, and resistance patterns. Antimicrob. Agents Chemother. 2011, 55, 2352-2361.

27. Raz, B.; Iten, M.; Grether-Buhler, Y.; Kaminsky, R.; Brun, R. The Alamar Blue assay to determine drug sensitivity of African trypanosomes (T. $b$. rhodesiense and T. $b$. gambiense) in vitro. Acta Trop. 1997, 68, 139-147.

28. Dardonville, C.; Fernandez-Fernandez, C.; Gibbons, S. L.; Jagerovic, N.; Nieto, L.; Ryan, G.; Kaiser, M.; Brun, R. Antiprotozoal activity of 1-phenethyl-4aminopiperidine derivatives. Antimicrob. Agents Chemother. 2009, 53, 3815-3821. 
29. Rodenko, B.; Van Der Burg, A. M.; Wanner, M. J.; Kaiser, M.; Brun, R.; Gould, M.; De Koning, H. P.; Koomen, G. J. 2,N6-disubstituted adenosine analogs with antitrypanosomal and antimalarial activities. Antimicrob. Agents Chemother. 2007, 51, 3796-3802.

30. Wallace, L. J. M.; Candlish, D.; De Koning, H. P. Different substrate recognition motifs of human and trypanosome nucleobase transporters. Selective uptake of purine antimetabolites. J. Biol. Chem. 2002, 277, 26149-26156.

31. LigPrep, version 2.5, Schrödinger Inc., LLC, New York, NY, 2011.

32. Mittal, R. R.; Harris, L.; McKinnon, R. A.; Sorich, M. Partial Charge Calculation Method Affects CoMFA QSAR Prediction Accuracy. . J. Chem. Inf. Mod. 2009, 49, 704-709.

33. Gaussian 03, R. C., M. J. Frisch, G. W. Trucks, H. B. Schlegel, G. E. Scuseria, M. A. Robb, J. R. Cheeseman, J. A. Montgomery, Jr., T. Vreven, K. N. Kudin, J. C. Burant, J. M. Millam, S. S. Iyengar, J. Tomasi, V. Barone, B. Mennucci, M. Cossi, G. Scalmani, N. Rega, G. A. Petersson, H. Nakatsuji, M. Hada, M. Ehara, K. Toyota, R. Fukuda, J. Hasegawa, M. Ishida, T. Nakajima, Y. Honda, O. Kitao, H. Nakai, M. Klene, X. Li, J. E. Knox, H. P. Hratchian, J. B. Cross, V. Bakken, C. Adamo, J. Jaramillo, R. Gomperts, R. E. Stratmann, O. Yazyev, A. J. Austin, R. Cammi, C. Pomelli, J. W. Ochterski, P. Y. Ayala, K. Morokuma, G. A. Voth, P. Salvador, J. J. Dannenberg, V. G. Zakrzewski, S. Dapprich, A. D. Daniels, M. C. Strain, O. Farkas, D. K. Malick, A. D. Rabuck, K. Raghavachari, J. B. Foresman, J. V. Ortiz, Q. Cui, A. G. Baboul, S. Clifford, J. Cioslowski, B. B. Stefanov, G. Liu, A. Liashenko, P. Piskorz, I. Komaromi, R. L. Martin, D. J. Fox, T. Keith, M. A. Al-Laham, C. Y. Peng, A. Nanayakkara, M. Challacombe, P. M. W. Gill, B. Johnson, W. Chen, M. W. Wong, C. Gonzalez, and J. A. Pople, Gaussian, Inc., Wallingford CT, 2004. 
34. Accelrys Software Inc., D. S. H., San Diego: Accelrys Software Inc., 2010.

35. SYBYL-X 1.2, T. I., 1699 South Hanley Rd., St. Louis, Missouri, 63144, USA. 
Table of Contents graphic

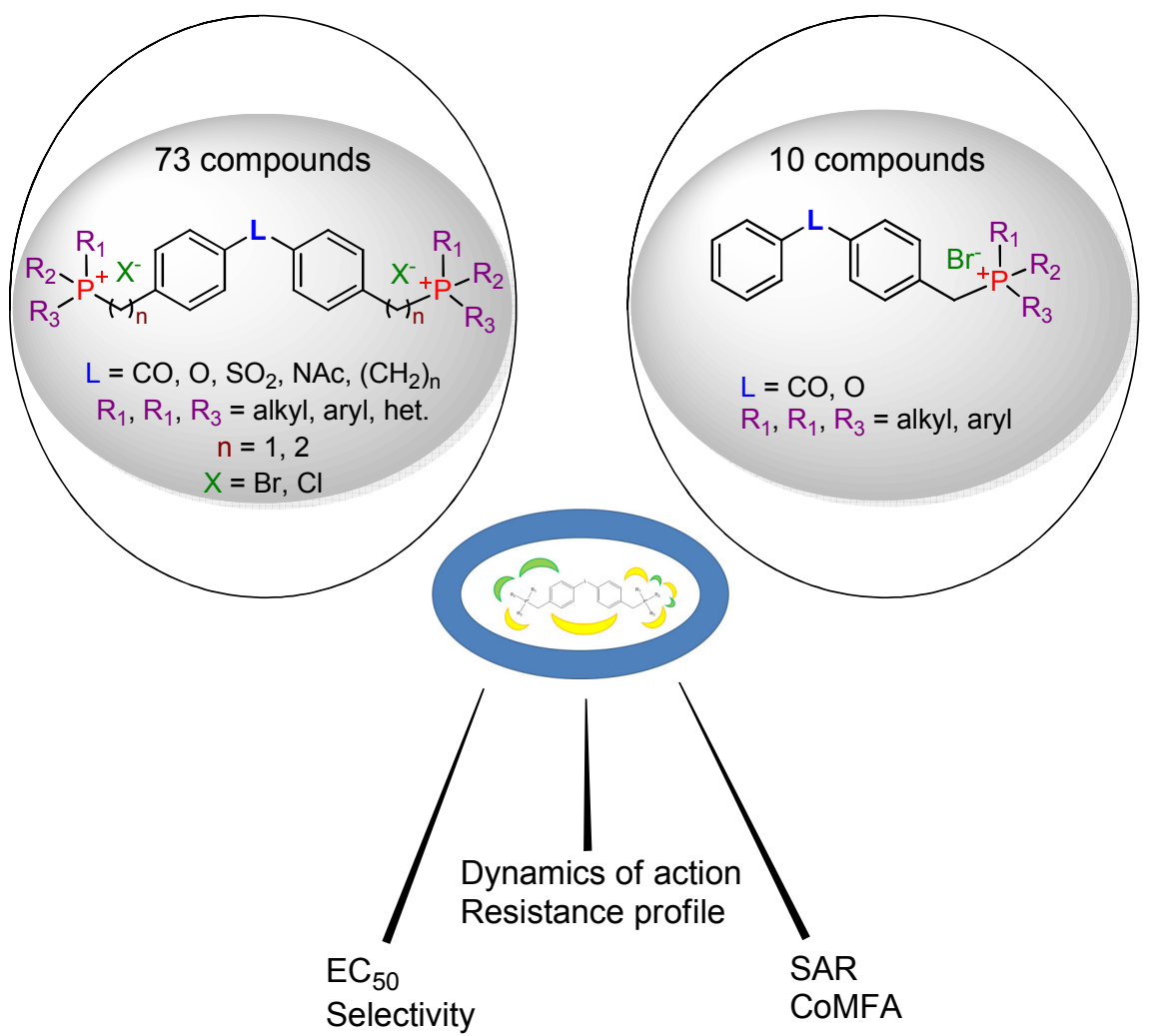

